\title{
Don Giovanni de' Medici - Artilleriegeneral in habsburgischen Diensten und kaiserlicher Festungsbaumeister: ein Beitrag zu seinen Leistungen als Architekturdilettant in Wien und den ungarischen Grenzgebieten
}

\author{
Mit elf Abbildungen
}

Don Giovanni de' Medici (1567-1621) war ein unehelicher Sohn von Cosimo I. de' Medici (1519-1574) und Eleonora degli Albizzi, weshalb ihm der Zugang zu politischen Ämtern verwehrt blieb. Ursprünglich war er - wie der kurz zuvor verstorbene Sohn Cosimos gleichen Namens - für eine kirchliche Karriere bestimmt, er interessierte sich jedoch für das Kriegshandwerk (Abb. 1), und so ging er 20jährig 1587-1589 nach Flandern, um an der Seite von Alessandro Farnese zu kämpfen, und anschließend 1594-1596 in den habsburgisch-türkischen $\mathrm{Krieg}^{1}$. In diesem Zusammenhang soll sein Interesse für die Festungsbau-

* Bei Zitaten werden Korrekturen am Rand oder Anfügungen oberhalb des Textes in spitze Klammern gesetzt $\langle>$; korrigierte bzw. durchgestrichene Wörter werden als solche getreu wiedergegeben; hier wiederholt verwendete Abkürzungen:

ASF Archivio di Stato, Firenze

M.d.P. Mediceo del Principato (Archivbestand des Florentiner Staatsarchives)

BNCF Biblioteca Nazionale Centrale di Firenze.

Ausdrücklich möchte ich hier Dr. Péter Farbaky vom Historischen Museum in Budapest für die Übersetzung einiger Namen ungarischer Festungen und Prof. Dr. Richard Bösel für wertvolle Hinweise bezüglich der Wiener Topographie und einiger ungarischer Ortsnamen danken. Sehr danken möchte ich auch dem Kunsthistorischen Institut Florenz für ein Stipendium, das mir ermöglichte, im Mai-Juni 2009 das Florentiner Aktenmaterial einzusehen.

${ }^{1}$ Für biographische Angaben vgl. E. BALOssI, Don Giovanni de' Medici - Saggio biografico. Torino 1899; vgl. auch G. Pieraccini, La stirpe de' Medici di Cafaggiolo: Saggio di ricerche sulla trasmissione ereditaria dei caratteri biologici, II. Firenze $1986\left({ }^{1} 1925\right), 217-$ 249. Verwiesen sei auch auf die von Cosimo Baroncelli, Giovannis langjährigem Sekretär 
kunst untersucht werden, insbesondere seine Rolle als Baumeister von Befestigungsanlagen unter Kaiser Rudolf II. Es wird auch auf seine persönlichen Begegnungen mit dem Kaiser in Prag einzugehen sein, insbesondere auf die Unterredungen am 23. Januar 1595 und Ende Januar 1596, als sie über die Befestigungsanlagen an der ungarischen Grenze sprachen und ihre Kenntnisse in Sachen Wehrbau austauschten. Grundlage meiner Untersuchungen ist ein Konvolut von Briefen von Giovanni de' Medici im Florentiner Staatsarchiv ${ }^{2}$, das bislang wenig untersucht worden ist, zumal aus der Sicht von Architekturhistorikern ${ }^{3}$.

Parallel dazu wird auch zu untersuchen sein, inwieweit in einer Epoche, in der italienische Bauweise und Kunst größte Anerkennung in Salzburg und anderen österreichischen Gebieten (wie auch sonst in weiten Teilen des Reiches) fanden ${ }^{4}$,

und Gehilfen, verfaßte ,Vita' (Discorso Istorico del sig $[$ no $]$ re Cosimo Baroncelli fatto à suoi figliuolj della vita e morte di Don Giovanni de Medici, figlio naturale del Gran Duca Cosimo Primo), die jüngst von Marina Macchio publiziert wurde: M. Macchio, Il Discorso del Sig.re Cosimo Baroncelli fatto a' suoi figliuoli dove s'intende la vita di Don Giovanni Medici [...]. Firenze 2009.

${ }^{2}$ Es handelt sich um ein Konvolut von ca. 400 Briefen, die in einem Band zusammengebunden worden sind (ASF, M.d.P., filza 5156); der Einband trägt folgende Aufschrift: L[ette]re d[e]ll'Ecc[ellentissi]mo s[ignor] Don Gio. Medici del[ l'] anno 1594 a tutto l'anno 1599: guerra d'Ungheria. Manche Briefe sind an den Cav. Belisario Vinta (1542-1613) gerichtet, der in diesen Jahren das Amt des Sekretärs und persönlichen Referenten des Großherzogs (sec [retari] o d [e]lser[enissi] mo gra $[n]$ Duca di Toscana) innehatte, die meisten jedoch an den Großherzog Ferdinand I. (reg. 1587-1608), seinen Halbbruder. Nicht alle Briefe sind Autographe von Don Giovanni, manche sind auch von seinem persönlichen Sekretär, Marco Giani, verfaßt und signiert (u. a. fol. 240, 242, 312, 429, 656-657, 733); einige Briefe sind von Cosimo Baroncelli, seinem Maggiordomo, geschrieben (u. a. fol. 110, 557 , 564-565, 587, 635-636, 661-662, 678-679). - Einige Briefe Don Giovannis sind in einem anderen Aktenschuber gelandet (ASF, M.d.P., filza 5154, fol. 202r [Briefe u. a. aus Strigonia vom August 1595], auch in: ASF, carte Alessandri, filza 10). Kopien der Antwortbriefe des Großherzogs, auf die Don Giovanni oftmals Bezug nimmt (u. a. fol. 309r: Tengo la amorevolissima lettera di V[ostra] A[ltezza] delli 3 stante), befinden sich - zusammen mit denen der Großherzogin und den Schreiben der Sekretäre - in ASF, M.d.P., filza 5153: Sie enthalten vor allem Äußerungen zur politischen Lage und zahlreiche Ermahnungen, ferner auch einige wenige anerkennende Bemerkungen bezüglich seiner Festungsentwürfe (s. u., Anm. 88).

${ }^{3}$ Die Briefe wurden z. T. schon von Ester Balossi und von Alessandro Gambuti herangezogen; vgl. BALOssi (wie in Anm. 1), bes. 24ff., 38f. (und im Dokumentenanhang am Schluß des Buches); vgl. A. Gambuti, L'altra architettura di Don Giovanni de' Medici, in: Scritti di storia dell'arte in onore di Roberto SAlvini. Firenze 1984, 455-460. Zum Teil wurden sie auch publiziert im Katalog Firenze e la Toscana dei Medici nell'Europa del Cinquecento. Firenze 1980, 94 (Nr. 47.7-10). Tatsache bleibt, daß die Briefe bislang vor allem in historischer Sicht ausgewertet wurden.

${ }^{4}$ Zu Salzburg vgl. insbesondere meine Dissertation: Der Salzburger Dom 1598-1630 - Unter besonderer Berücksichtigung der Auftraggeber und des kulturgeschichtlichen Umfeldes. Weimar 1999 (Diss. Univ. Bonn 1992), bes. 67ff., 89ff. Zur Verbreitung der Renaissance in 
die italienische Militär- und Festungsbaukunst rezipiert und akzeptiert wurde. Oder ob man bei den Festungsbauten eine leicht distanzierte Haltung gegenüber den aus Italien importierten Neuerungen erkennen kann, wie dies beim Wiener Neugebäude der Fall gewesen ist, wo gezielt italianisierende Motive verwendet wurden, zugleich aber auch traditionell einheimische Elementes.

\section{Die Beziehungen Habsburg-Medici und die Türkenkriege ENDE DES 16. JAHRHUNDERTS}

Mehrere Heiraten zwischen den Familien Medici und Habsburg, so die von Duca Alessandro de' Medici (reg. 1530-1537) mit Margarete von Parma 1536, von Großherzog Francesco de' Medici (reg. 1574-1587) mit Johanna von Österreich (1548-78), eine 1565 mit großem Aufwand gefeierte, aber sehr unglücklich verlaufene Ehe 6 , und später zwischen Großherzog Cosimo II. de’ Medici (reg. 1609-1621) und Maria Magdalena von Habsburg (1589-1631) ${ }^{7}$, sollten zur Festigung des seinerzeit zwischen Kaiser Karl V. und den Medici geschlossenen Bündnisses führen. Bekanntlich hatte Kaiser Karl V. die Medici als Vasallen eingesetzt, und durch ihn hatten sie 1532 den Herzogstitel bekommen. Die im Februar 1535 in Neapel arrangierte Heirat von Duca Alessandro de' Medici (15101537) mit Margarete von Parma, in Italien korrekterweise Margherita d'Austria genannt, einer unehelichen Tochter Karls V., war ein erster Versuch, das Haus Medici enger an den kaiserlichen Machtbereich zu binden ${ }^{8}$.

Kärnten vgl. B. Kienzl-W. Deuer. Renaissance in Kärnten (Dic Kunstgeschichte Kärntens). Klagenfurt 1996. Vgl. auch G. SKALECKI, Deutsche Architektur zur Zeit des Dreißigjährigen Krieges - Der Einfluß Italiens auf das deutsche Bauschaffen. Regensburg 1989.

${ }^{5}$ Hierzu vgl. meinen Beitrag: Il »Neugebäude « di Vienna - Genesi e analisi di un insolito complesso. Annali di architettura 18-19 (2006-07), 143-168, bes. $154 \mathrm{f}$.

${ }^{6}$ Zumindest seit 1574 ist die Geliebte Bianca Cappello (1548-87) in der Nähe des Großherzogs nachweisbar. Zur Fahrt von Großherzog Francesco I. nach Innsbruck und Wien sowie anschließend nach München und Prag und den Beziehungen des Großherzogs zu den Habsburgern vgl. L. Zangheri, L'Arciduca Ferdinando del Tirolo e i Medici, in: Musagetes - Festschrift für Wolfram Prinz zu seinem 60. Geburtstag am 5. Februar 1989, hrsg. von G. KecKs. Berlin 1991 (Frankfurter Forschungen zur Kunst 17), 347-359, bes. 351.

${ }^{7}$ Anläßlich des frühzeitigen Todes des Großherzogs bemerkt der Historiker Francesco Settimanni (1681-1763): Era questo Principe delicatissimo di complessione ed essendosi accompagnato coll'Arciduchessa M[ari] a Maddalena d'Austria di età di anni 12 ed avendo procreato molti figliuoli di essa, si era indebolito lo stomaco di maniera che non gli faceva piu l'ufficio suo; onde cominciosi ad ammalare l'anno 1614. [...] e di corpo tanto delicato messo a canto alla moglie, si era strutto e ridotto in $\langle$ tanto $\rangle$ cattivo stato (ASF, Ms. 132, fol. 608r: Eintrag unter Datum 28. 2. 1621).

${ }^{8}$ BNCF, Palatino 900 (Varie notizie prese alla giornata di fatti memorabili seguiti nella città di Firenze dall'anno 1557 fino all'anno 1591), 136:...p [er ] via di regali dati a Ministri dell'Imperadore acciò gli favorisse, il di 29 di Febbrario 1535 giorno di Carnevale dette in Napoli 
Bevor aber Karl V. Herzog Cosimo in seinem Amt bestätigte, entsandte er einen Botschafter nach Florenz, um die Stimmung in der Stadt zu überprüfen, zumal der Vorgänger Alessandro durch einen Familienangehörigen ermordet worden war. Angesichts der unklaren politischen Lage beschloß der Botschafter - sicherlich im Einverständnis mit dem Kaiser -, Cosimo zwar in seinem Amt als Herzog zu bestätigen, ihm aber vorsichtshalber nicht die Schlüssel der Festungen auszuhändigen?. In der Folgezeit haben wir es mit einer Politik des gegenseitigen Mißtrauens zu tun. Cosimo seinerseits betonte - zumal gegenüber italienischen Herrschern - die Unabhängigkeit des Florentiner Staates (damals noch ohne die Gebiete von Siena) ${ }^{10}$, andererseits ließ er keine Gelegenheit aus, dem Kaiser zu huldigen - so in Genua im August 1541 ${ }^{11}$; auch wurden anläßlich jeder Kaiserwahl, Geburt eines Thronnachfolgers oder Hochzeit der Kaiserfamilie Botschafter entsandt und in Florenz Festlichkeiten organisiert ${ }^{12}$. Zudem unterstützte er

l'anello a Madama Margherita d'Austria, figlia naturale di sua M[aestà] C[esarea] dotando di $200 \mathrm{~m}$ [ila] fiorini d'oro e in quella sera fu fatto un bellissimo convito.

9 BNCF, Palatino 900 (wie in Anm. 8), 171f.: Ferdinando de' Silva detto il Conte Sifonte, Ministro di Carlo $V$ in Italia e suo Ambasciadore [...], venne in Firenze [...]; questo per scoprire gli umori e le passioni de' cittadini, come in favore che in disfavore di Cosimo. Tenne diverse pratiche e vedendo tante varietà di cervelli alli 21 Giugno $p$ [er] autorità concessagli da Sua Maestà Cesarea dichiarò il Principato fosse caduto nel sig [no $] r$ Cosimo, come più prossimo e di maggior età di alcun'altro della detta Casa de' Medici, e ne' suoi successori discendenti leggittamente e costitui il detto sig [no]r Cosimo primo Capo della Repubblica e Stato Fiorentino, e se ne fece pubblico stromento socritto dal detto Conte e sigillato col suo sigillo, facendo giurare al sig[no]r Alessandro Vitelli di tener la Fortezza a nome dell'Imperadore, che lo fece senza guardare quel che dicesse il Duca o gli altri.

${ }^{10}$ Wie bereits Luigi Carcereri in seiner Monographie von 1926 herausstrich, betrachtete Cosimo sein Territorium als unabhängig vom Kaiserreich, ja ihm nicht einmal untertan; vgl. L. Carcereri, Cosimo primo Granduca, Teil 1: 1560-1565. Verona 1926, 189ff. Ebenso streicht Danilo Marrara in seinem Beitrag von 1980 heraus, daß der Botschafter Averardo Serristori erklärt habe, daß Cosimo nicht einmal politische Beziehungen zum Reich hätte (essere il Duca principe assoluto e non avere relazioni con lo Impero); vgl. D. MARrara, I rapporti giuridici tra la Toscana e l'Impero (1530-1576), in: Firenze e la Toscana dei Medici nell' Europa del '500 (Atti del convegno internazionale, Firenze, 9-14 giugno 1980). Firenze 1983, 119.

${ }^{11}$ Als Quelle diente die vom Historiker Francesco Settimanni (1681-1763) chronologisch geordnete Sammlung historischer, kulturpolitischer und auch sozialwissenschaftlicher Fakten der Medici-Zeit, die unpubliziert ist (Memorie fiorentine: ASF, Ms. 125-147); bes. ASF, Ms. 126, fol. 226r: L'Imperatore Carlo Quinto giunse a Genova, dove fu incontrato e visitato dal Duca Cosimo de' Medici, che era da $S[$ ua] $M[$ aestà $]$ fu molto accarezzato e ben trattato (Eintrag unter Datum August 1541). Zur Person Settimannis vgl. G. Benedetti, Notizie e documenti intorno la vita di Francesco Settimanni fiorentino e cavaliere di S. Stefano. Firenze 1875.

${ }^{12}$ Besonders unter Großherzog Francesco I. de' Medici dokumentiert: so im Fall der Krönung Rudolfs II. am 28. 11. 1575: ... fuochi e grandi allegrezze $p$ [er] l'incoronazione di Ridolfo in Re de Romani (F. Settimanni, Memorie fiorentine IV [= ASF, Ms. 129], fol. 
Karl V. militärisch wie auch finanziell, so in den Auseinandersetzungen mit Herzog Wilhelm V. von Kleve $(1543)^{13}$ und 1548 im Schmalkaldischen Krieg, als er ein Kontingent von 300 Reitern sandte ${ }^{14}$.

Der Kaiser wiederum antwortete zurückhaltend, indem er Cosimo zwar weitere Festungen überließ, ohne ihm jedoch die Vollmacht über alle militärischen Stützpunkte in der Toskana zu geben (so überließ Karl V. Cosimo Portoferraio auf Elba, nicht aber die südlichen Gebiete der Insel, und später die Gebiete von Siena, nicht aber die Festungen vom Argentario und von Piombino). Einige finanzielle Transaktionen zugunsten des Kaisers hatten den Zweck, ihm endlich wichtige Festungen wie Florenz und Livorno abzutrotzen und sie endgültig Cosimo zu übergeben $(1543)^{15}$.

Das gegenseitige Mißtrauen bzw. geringe Vertrauen hatte sicherlich verschiedene Gründe: Zum einen lag es an den historischen Ereignissen der letzten Jahrzehnte und Jahrhunderte, aber auch an den schwachen wirtschaftlichen und kulturellen Beziehungen zwischen den beiden Ländern. Während mit Venedig trotz einiger kriegerischer Auseinandersetzungen in dieser Epoche ${ }^{16}$ - und mit Genua stets ein intensiver wirtschaftlicher Austausch stattfand (man denke nur an den Fondaco dei Tedeschi in Venedig), gelang es den Florentinern niemals, ihre Waren, speziell ihre (Woll-) Tücher, in den deutschsprachigen Raum zu verkaufen; hier dominierten eindeutig die flämischen Handelshäuser ${ }^{17}$. Ein Problem

49v) und der Wahl Kaiser Maximilians II. zum König von Polen am 24. 12. 1575: Tutto il giorno fu suonato a festa e la sera fuorno fatti i fuochi $[$ [er] la nuova che l'Imperator Massimiliano era stato eletto Re di Pollonia (ebd., fol. 50r). Anläßlich der Hochzeit von König Philipp II. mit Margarete von Österreich reiste Don Giovanni 1598/99 nach Madrid.

${ }^{13}$ Vgl. Settimanni, Memorie fiorentine II/1 (= ASF, Ms. 126), fol. 281v: L'Imperatore trovandosi in gran necessità di denari $p[e r]$ far guerra al Duca di Cleves ricevette a Trento 155 mila scudi dal Duca di Firenze (Eintrag unter Datum Juli 1543).

${ }^{14}$ Vgl. ebd., fol. 358: Avendo risoluto l'Imp[erator] e di muovere la guerra nella Germania a Principi Protestanti, il Duca Cosimo gli mando in ajuto piu di 300 cavalli sotto la cura di Ridolfo Bastiani, i quali a Bologna si unirono con $12 \mathrm{~m}$ [ila] fanti Italiani mandati $p$ [er] lo med [esim] o effetto dal Papa (Eintrag unter Datum 18.7. 1546). Auch im Juli 1566: ... nella sovvenzione data all'Imp[erator]e di ducati $200 \mathrm{~m}$ [ila], nella spedizione al med[esimo] Imp [erator $]$ e di tremila fanti; vgl. F. Settimanni, Memorie fiorentine III (= ASF, Ms. 128), fol. 371v.

${ }^{15}$ Vgl. Settimanni, Memorie fiorentine II/1 (= ASF, Ms. 126), fol. 322: Il Duca Cosimo restitui a Genovesi scudi $80 \mathrm{~m}[$ ila] che da loro aveva preso in prestito [...] in occasione che avendo ricuperate dall'Imperatore le Fortezze di Firenze e di Livorno aveva dovuto dare a $S[u a] M[a e s t a]$ Cesarea una grossa somma di denaro (Eintrag unter Datum Okt. 1544).

${ }^{16}$ In die Jahre 1592-1617 fiel u. a. der Uskokken-Krieg.

${ }^{17}$ Vgl. P. Malanima, L'industria fiorentina in declino fra Cinque e Seicento - linee per un'analisi comparata, in: Firenze e la Toscana dei Medici, Atti (wie in Anm. 10), 295-308, bes. 297. Die flämische und holländische Wollware drang bis auf den venezianischen Markt vor und von dort aus weiter in den Mittelmeerraum; vgl. C. WirTz, Köln und 
stellten die Sprachbarrieren dar, sicherlich auch das Fehlen einer eigenen Handelsflotte, da Großherzog Cosimo erst um 1548 damit begann, eine eigene Flotte von Handels- und Kriegsschiffen aufzubauen ${ }^{18}$. In diesem Zusammenhang sei auch erwähnt, daß es der 1443 wieder eröffneten Universität Pisa ${ }^{19}$ nicht gelang, Studenten aus dem deutschsprachigen Raum anzuziehen. Die deutschen Studenten bevorzugten, wohl auch wegen der Tanz- und Reitschulen, vornehmlich Siena (und dies auch noch nach der Einnahme der Stadt seitens der Florentiner $1555)^{20}$.

Aber auch auf kulturellem Gebiet war der Austausch recht schwach. Viele Künstler und Musiker sind seit Dürer und Heinrich Schütz nach Venedig ${ }^{21}$ oder nach Genua gekommen ${ }^{22}$, doch nur sehr wenige deutsche und österreichische Künstler zog es bis nach Florenz, zumal im 16.-17. Jahrhundert. An den Hof der Medici kamen hingegen viele Flamen: so der zu großem Ruhm gelangte Bildhauer Giambologna und die Maler Justus Sustermans und Justus Utens ${ }^{23}$. Auch

Venedig - Wirtschaftliche und kulturelle Beziehungen im 15.-16. Jahrhundert (Beihefte zum Archiv für Kulturgeschichte 57). Köln-Weimar-Wien 2006 (Diss. Univ. Zürich 2001), 251.

${ }^{18}$ Vgl. Settimanni, Memorie fiorentine II/1 (ASF, Ms. 126, fol. 422v): Il Duca Cosimo trovandosi in Livorno fece mettere in mare la $p$ [ri] ma Galea che aveva fatta fabbricare, detta la Pisana (Eintrag unter Datum 10. 3. 1548); vgl. auch G. G. GUARnieri, Cavalieri di Santo Stefano - contributo alla storia della Marina Militare Italiana (1562-1859). Pisa 1928. Das Fehlen einer eigenen Flotte erklärt eventuell auch, daß die Florentiner Handelshäuser bei den Festumzügen in Brügge stets nur an dritter oder gar an vierter Stelle, hinter den venezianischen und Genueser Kollegen und manchmal auch hinter den Lucchesischen Handelsvertretern, postiert wurden.

${ }_{19}$ Zur Stärkung der Universität wurde u. a. den Florentiner Studenten untersagt, außerhalb von Pisa zu studieren.

${ }^{20}$ Angeblich stammten nur 6\% der Studentenschaft aus dem hiberischen und nordalpinen Kulturraum; vgl. C. B. Schmit, The Studio Pisano in the European Context of the Sixteenth Century, in: Firenze e la Toscana dei Medici, Atti (wie in Anm. 10), 24. Die Vorliebe der nordalpinen Völker für die Universität Siena erklärt F. Settimanni folgendermaßen (ASF, Ms. 132, fol. 215v): Nella città di Siena pratica assai la Nazione Tedesca ed altre oltramontane, quali si vengono $p[$ er $]$ imparare la lingua, il cavalcare ed altre virtu cavalleresche e sono d'utilità grande alla città, e quegli hanno i loro Privilegi, esenzioni ed ordini sempre osservabili (Eintrag Juli 1614).

${ }^{21}$ Erwähnt seien hier nur Adam Elsheimer, der ca. 1598-1600 in Venedig weilte, Hans Rottenhammer (1589-1606 in Venedig) und Christoph Schwarz, von dem Ridolfi schreibt, er sei ein "Schüler Tizians“.

${ }^{22}$ So Josef Furttenbach d. Ä., der dort ca. 1609-1616 blieb.

${ }^{23}$ Erwähnt sei auch der flämische Steinschneider Jan Flach (bzw. Flasch?), der zunächst am Hof der Medici als Falkner eine Anstellung hatte und erst seit 1585 als Gehilfe bei Steinschneidern arbeitete. Zu dem um 1654 verstorbenen Künstler vgl. P. URBANI, Repertorio, in: Ferdinando I de' Medici 1549-1609 - maiestate tantum. Ausstellungskatalog, hrsg. von M. Bietti-A. Giusti. Livorno 2009, 145f. Zu den in Florenz tätigen Flamen zähl- 
wenn zumindest ab 1574 und insbesondere ab 1590 ein reger Besuch bayerischer und Württemberger Herzöge sowie Habsburger-Prinzen in Florenz nachweisbar ist $^{24}$ (und der Münchner Perseus-Brunnen 1581/82 mit Materialien angefertigt wurde, die Großherzog Francesco aus Florenz zusandte), änderte sich an der ursprünglichen Situation wenig, d. h. weiterhin traten auffallend wenige deutschsprachige Künstler eine Reise nach Florenz an. Als eine Ausnahme kann der Maler Johann Karl Loth (1632-1698) angesehen werden, der zumindest ab 1691 für den Prinzen Ferdinand III. von Toskana arbeitete.

Intensiver scheinen hingegen die Kontakte auf militärischem Gebiet gewesen zu sein, zumindest seit 1541, als Cosimo sich eine persönliche Garde von zunächst 200 deutschen und wohl auch Schweizer Soldaten zulegte, die von einem Fugger angeführt wurde: Entrò in Firenze una Guardia di Tedeschi in n[umer]o di 200 fanti sotto la condotta di Baldassar Fuggher loro comandante, a quali fu dato luogo nella nuova Cittadella ${ }^{25}$. Bereits zwei Jahre später (1543) stockte Cosimo die Truppen auf 1500 Mann auf ${ }^{26}$.

ten auch der aus Deventer stammende Kupferstecher Adriaen Haelwegh (ca. 1637-1702) sowie der Naturwissenschaftler Gerhard Dorn, der sich als Interpret der Ideen von Paracelsus einen Namen gemacht hatte: als Autor der Publikation Aurora thesaurusque philosophorum Theophrasti Paracelsi (Basel 1577). Er hatte anscheinend für Don Antonio de' Medici, den unehelichen Sohn von Großherzog Francesco, eine italienische Übersetzung eines Traktates (Anatomia delli corpi vivi) verfaßt. Das Manuskript (BNCF, Palatino 666) befand sich beim Tod Don Antonios in dessen Haus. Es sollte wohl ursprünglich publiziert werden; vgl. Firenze e la Toscana dei Medici, Ausstellungskatalog (wie in Anm. 3), 182 (Nr. 5.54).

${ }^{24}$ Von den Habsburgern kamen nur im November 1581 Erzherzog Maximilian (15581618) und 1598 der zukünftige Kaiser Ferdinand II., damals noch Erzherzog, nach Florenz. Von deutscher Seite kamen offenkundig vor allem die Herrschaftshäuser südlicher Regionen: Im Mai 1574 traf der Sohn des Herzogs von Bayern in Florenz ein, Wilhelm V. von Wittelsbach (1548-1626); im November 1583 kam der Duca di Bruswich (= Brunswick [Braunschweig]). Nachdem 1591 der bayerische Botschafter in Florenz eingetroffen war, gelangten 1592 die Söhne des bayerischen Herzogs Wilhelm von Wittelsbach und schließlich 1593 Kurfürst Maximilian in offizieller Mission nach Florenz, der nochmals 1597 die Stadt besuchte. Im Januar 1600 ist Herzog Friedrich I. von Württemberg kurz in Florenz gewesen (incognito).

${ }^{25}$ Settimanni, Memorie fiorentine II/1 (= ASF, Ms. 126), fol. 228: Demnach wurden sie in der Festung (Fortezza da Basso) untergebracht. Diesem Vorbild folgte wenige Jahre später auch Genua, das sich eine ähnliche Wachttruppe zulegte.

${ }^{26}$ Ebd., fol. 287v: Giunse in Firenze una Banda di $\langle 1500\rangle$ Tedeschi fatta venire dal Duca Cosimo, de quali una parte fu messa a guardia del Palazzo di Piazza [...], un'altra parte entró in Castello ed il rimanente fu mandato verso Pisa (Eintrag unter Datum 12.8. 1543): Durch die „Lanzi“ bekam die Loggia auf der Piazza della Signoria ihren Namen. Sie hatten eine eigene Kapelle in Florenz, die Cappella di S. Barbara. 
Während Cosimo - zumal nach $1553^{27}$ - eine klare Position gegen Frankreich bezog und sich prinzipiell als treuer Alliierter des Kaisers erwies ${ }^{28}$, haben seine Nachfolger, vor allem Großherzog Ferdinand I. (1587-1608), gegenüber der französischen Krone eine sehr viel konziliantere Politik verfolgt ${ }^{29}$. Zwar ließ Ferdinand einige Inseln vor der Hafeneinfahrt von Marseille 1597 besetzen und befestigen $^{30}$, um den französischen Schiffen im Falle eines Krieges die Ausfahrt aus dem Hafen zu behindern, doch schließlich einigte man sich gütlich und beschloß eine Heirat ${ }^{31}$. Folglich kam es zu einer Abkühlung der Beziehungen zwischen den Habsburgern und den Medici ${ }^{32}$.

In einem Punkt folgte Ferdinand I. der Politik Cosimos: Wie dieser unterstützte auch er den Kaiser Rudolf II. in kritischen Momenten mit seinen Truppen. Bereits 1577/78 hatte sein Vorgänger, Großherzog Francesco (reg. 15741587), ein Kontingent von Soldaten und Technikern entsandt, um demselben Kaiser in den Türkenkriegen beizustehen ${ }^{33}$. Für eine weitere Kampagne des türkisch-ungarischen Krieges, in den Jahren 1594-1596, stattete Ferdinand die Truppe nicht nur großzügig aus, auch der persönliche Einsatz seitens der Angehörigen der Medici-Familie war entsprechend groß ${ }^{34}$. Dieser Krieg und speziell die

${ }^{27}$ Settimanni, Memorie fiorentine II/2 (= ASF, Ms. 127), fol. 625: Parendo al Duca Cosimo di essere assai chiaro dell'animo del Re di Francia contrario a lui, essendo stato prima neutrale nelle <turbolenze > di Siena, comincio a pendere dalla parte Imperiale (Eintrag unter Datum Dezember 1553).

${ }^{28}$ In diesem Sinne ließ sich Erzherzog Ferdinand von Tirol 1575 die Rüstung Cosimos nach Ambras senden: ... saria peró molto grato d'haver l'armadura, che soleva portare il S[igno]r Padre di V[ostra] E[ccellenza], felice memoria, come soldato famoso [...] nelle imprese commesseli dal Imperatore Carlo, felice memoria, nelle guerre contra li suoi inimici et disobedienti; vgl. Zangheri, L'Arciduca Ferdinando (wie in Anm. 6), 347-359, bes. 356 (Dok. Nr. 1).

${ }^{29}$ Vgl. F. Diaz, Il Granducato di Toscana - I Medici (Storia d'Italia XIII/1). Torino 1976, 285-287.

${ }^{30}$ Vgl. Balossi (wie in Anm. 1), 41f.; vgl. auch C. Promis, Biografie di ingegneri militari dal secolo XIV alla metà del XVIII. Torino 1874 (Miscellanea di storia italiana 14), 759f.

${ }^{31}$ Die Heirat zwischen Maria de' Medici und König Heinrich IV. von Frankreich (1600). Dies war letztendlich auch schon unter Cosimo geschehen, als Caterina de' Medici (1519-1589), aus einer Seitenlinie der Familie stammend, den französischen König Heinrich II. 1547 heiratete, wodurch beide Familien verwandt waren.

${ }^{32}$ Die Korrespondenz wurde nunmehr vor allem von der Gattin des Erzherzogs, einer gebürtigen Gonzaga, ebenfalls mit den Medici verwandt, aufrechterhalten; vgl. ZANGHERI, L'Arciduca Ferdinando (wie in Anm. 6), 347-359, bes. 351-354.

${ }^{33}$ Diesem Kontingent gehörte u. a. der Ingenieur und Festungsexperte Antonio Lupicini an; vgl. L. A. Maggiorotti, Architetti e architetture militari, II (L'opera del genio italiano all'estero, serie 4a : Gli architetti militari). Roma [1936], 110, 183, 437.

${ }^{34}$ Vgl. T. von BodgaY, Grundzüge der Geschichte Ungarns. Darmstadt ${ }^{4} 1990$. Vgl. auch G. MARRI, La partecipazione di don Giovanni de' Medici alla guerra d'Ungheria (1594-95 e 1601). Archivio storico italiano 99 (1941), 50-59. 
Leistungen von Don Giovanni de' Medici werden im Folgenden Gegenstand meiner Untersuchen $\operatorname{sein}^{35}$.

\section{Don Giovanni De' Medici In Ungarn}

Ende Juni 1594 verließ Don Giovanni zusammen mit 2000 Soldaten (fanti) die Stadt Florenz in Richtung Ungarn. Seinen Bestallungsbrief, die sog. patente, wodurch ihn der Kaiser im Amt eines Generals der Artillerie (Generale dell'Artiglieria) bestätigte, hatte er wenige Tage zuvor erhalten, als er sich noch in Florenz aufhielt ${ }^{36}$. Die Reiseroute führte über Bologna, Mantua und Innsbruck, wo er einen kurzen Halt einlegte ${ }^{37}$. Zunächst reiste er nach Regensburg, um Kaiser Rudolf II. zu sprechen ${ }^{38}$. Anschließend begab er sich nach Wien und dann direkt an die ungarische Front, an der er am 4. August eintraf (ein zweites Heereskontingent unter der Leitung von Don Antonio de' Medici, dem unehelichen Sohn von Großherzog Francesco de' Medici, traf am 16. August in Wien ein) ${ }^{39}$.

${ }^{35}$ Untersuchungen liegen bislang von E. Balossi (1899), A. Gambuti (1984) und D. Landolfi (1988) vor; vgl. BALOSsI (wie in Anm. 1), 24ff. und 93-95 (Dok. Nr. 6); GAmbuti (wie in Anm. 3), 455-460; D. Landolfi, Don Giovanni de' Medici 'principe intendente in varie scienze'. Studi secenteschi 29 (1988), 125-162.

${ }^{36}$ Das Dokument hat sich im Nachlaß von Don Giovanni erhalten (ASF, carte Alessandri, filza 10, fol. 42-50 [einst fasc. 6]): Patente dell'Imperatore Rodolfo II per la nomina di Giovanni de' Medici a 'Generale di artiglieria', copia tedesca del 1594, mit beiliegender lateinischer Übersetzung aus der Zeit und nachträglicher italienischer Übersetzung. Wie Don Giovanni in einem Brief vom 10.2. 1596 festhält, hatte er allein die oberste Befehlsgewalt über die Artillerie: $S[u a]$ Maesta [...] oltre a quello che mi disse nella prima audienza, $m$ 'ha replicato che d [ el]l'onore et reputatione di mia $p[$ er $]$ sona havrebbe particolare cura e pensiero, con havermi accertato che d [e]lla carica data di generale d [el] l'Artig [leri] a ad altri in Ungheria superiore non haveva saputo cosa alcuna (ASF, M.d.P., filza 5156, fol. 749r). Sehr zum Ärger von Don Giovanni hatte angeblich auch der ehem. Oberst der Florentiner Landsknechte, Ferrante Rossi, 1595 eine ähnliche Vollmacht bekommen: Ho inteso da un Cap [itan]o mandato qui dal sig.r Ferrante Gonzaga che il s[ignor] Ferrante Rossi habbi havuto da $S[u a] M[a e s]$ tà patente di Ge [ner] ale dell'Artiglieria in Hungheria superiore, se questo è V [ostra] A[ltezza] vede come mi viene scemata la mia Patente (ASF, M.d.P., filza 5156, fol. 612r; vgl. auch ebd., fol. 737r: zit. in Anm. 43).

${ }^{37}$ Briefe von Don Giovanni sind aus Bologna (vom 6. 7. 1594), Mantua (vom 7. 7. 1594), Trient (vom 9.7. 1594) und auch aus Innsbruck (12.7. 1594) erhalten. In Innsbruck traf er zunächst auf den Marchese di Borgaut, anschließend auch den Erzherzog Ferdinand von Tirol, mit dem er eine dreistündige Unterredung hatte: ... ho visitato il s[igno] $r$ Arciduca Ser [enissi $]$ mo essendo stato seco in discorso bene 3 hore (ASF, M.d.P., filza 5156, fol. 23r). Für weitere Briefe siehe ASF, M.d.P., filza 4459; vgl. ZANGHERI, L'Arciduca Ferdinando (wie in Anm. 6), 347-359, bes. 355, Anm. 19.

${ }^{38}$ ASF, M.d.P., filza 5156, fol. 47 (Brief vom 21.7. 1594): ... sono stato visto dalla Maestà d [el]l'Imp [erator] e begnissimamente et onorato in tutte le maniere.

${ }^{39}$ Ein erster Brief ist von Don Giovanni aus Györ bzw. Raab vom 11. 8. 1594 erhalten (ASF, M.d.P., filza 5156, fol. 91r). - Don Antonio de' Medici hatte mit einem Heer von 100 
Der erste Kontakt mit den österreichischen und deutschen Truppen und ihren Vorgesetzten scheint herzlich gewesen zu sein: ... sendo stato ricevuto et onorato in tutte le maniere dal Ser [erenissi $]$ mo s $[$ igno $]$ r Arciduca ${ }^{40}$, berichtet er in einem Brief. Allerdings kamen bald erste Schwierigkeiten, insbesondere häuften sich Klagen über das Wetter (Qua si travaglia et se bene $p[$ er $]$ il clima non ci sono le satisfattioni a pieno, tuttavia l'essere ben trattato [...] ricompensa ogni $\left.\cos a^{41}\right)$, aber auch Mentalitätsunterschiede machten sich bemerkbar: Questi tedeschi sono una gente goffa malogna, ma con il tempo ci affaremo insieme $e^{42}$. Der Alltag war zudem von Verständigungsschwierigkeiten gekennzeichnet ${ }^{43}$. Zudem nehmen Berichte über den schlechten gesundheitlichen Zustand der Truppe zu (Le malattie qua ci consumano molta gente ${ }^{44}$ ) - vor allem scheint Typhus grassiert zu haben: Tutti coloro che hanno qua febbre si scuoprano le petecchie et Curtio Guglielmi ne è tutto pieno ${ }^{45}$. Die militärisch ungünstige Lage - anfängliche Erfolge konnten da wenig helfen führte dazu, daß viele Soldaten des italienischen Kontingents, speziell nach einem Jahr, desertierten, um nach Italien zurückzukehren: ... dicendosi $p[$ er $]$ ognuno che li italiani non arrivino a 2000 et hoggi che è venuto di la [fol. 665r] dal lor quartiere

Kürassieren (corrazzieri) und 100 Schützen (archibusieri) erst Mitte Juli Florenz verlassen (ASF, M.d.P., filza 5132, fol. 24-25). Im Gegensatz zu Don Giovanni kehrte er (nach einer Verletzung im September 1594) bereits Ende November nach Florenz zurück, um dann nochmals im August 1595 für vier Monate nach Ungarn zu kommen (Rückkehr in Florenz am 1. Januar 1596 dokumentiert); vgl. P. F. Covoni, Don Antonio de' Medici al Casino di San Marco. Firenze 1892, 46ff. Vgl. auch F. Lutr, Don Antonio de' Medici e i suoi tempi. Firenze 2006 (Quaderni della Fondazione Carlo Marchi per la Diffusione della Cultura e del Civismo in Italia 27), $111 \mathrm{ff}$.

${ }^{40}$ ASF, M.d.P., filza 5156, fol. 91r: Brief vom 11.8. 1594.

${ }^{41}$ ASF, M.d.P., filza 5156, fol. 94r: Brief vom 18. 8. 1594 an die Großherzogin von Toskana.

${ }^{42}$ ASF, M.d.P., filza 5156, fol. 77r: Brief vom 11. 8. 1594. Die Kritik stieß in Florenz auf wenig Freude, denn sofort bekam er postwendend eine entsprechende Belehrung - und zwar von der Großherzogin, in Form eines 'inserto' : ... si come scrivono qua pubblicamente cosi debbon dir costi molto male della natione todesca et questo non è punto a proposito et non può se non cagionar odio et detrimento contro li Italiani et la natione predominante nel luogo dove si serve si deve sempre honorarla (ASF, M.d.P., filza 5153, fol. 8r: Brief vom 23. 8 . 1594). Noch kritischer äußert sich Don Giovanni in einem Brief vom 19. Sept. 1595: ... questi e altri disordini nascono tutti; parte $p[$ er $]$ mancamento di denari, parte $p[e r]$ negligenza e lunghezza solita de Todeschi, e forse ancora $p[e r]$ l'invidia che si ha alli Italiani del honore e aquisto di questo luogo (ASF, M.d.P., filza 5156, fol. 548v-549r).

${ }^{43}$ Gelegentlich erwähnt Don Giovanni Verständigungsprobleme: ... havendomi esposto in nostra lingua la sostanza di essi che era in tedesco, lo pregai a mandarmela in Italiano (ASF, M.d.P., filza 5156, fol. 333r). Ho visto finalmente in viso la patente del s[ignor] Ferrante $d[e$ ?] Rossi traslata in Italiano (ASF, M.d.P., filza 5156, fol. 737r).

${ }^{44}$ ASF, M.d.P., filza 5156, fol. 237r: Brief Don Giovannis vom 4. 11. 1594.

${ }^{45}$ ASF, M.d.P., filza 5156, fol. 242v: Brief von Marco Giani vom 11. 11. 1594. Er hatte Don Giovanni als Vermesser und Zeichner von Plänen gedient (s. u., Anm. 103 und 104). 
il Cav [alie] re Dati dice affermativamente che in modo nessuno non son rimasti 500 soldati, et che ogni giorno se ne fuggono ${ }^{46}$.

\section{Don Giovannis Anstellung als Kaiserlicher Festungsbaumeister IN WIEN UND UNGARN}

In einem Brief vom 30. November 1594 aus Wien erfahren wir von der Anstellung Don Giovannis als oberster kaiserlicher Festungsbaumeister, ein sehr ehrenvolles und verantwortungsvolles Amt: Quanto al carico che mi impone Sua Maestà $d[e]$ lla sopraintendenza $d[e]$ lla fortificazione di Vienna et $d[e] l l i$ luoghi dell'Ungheria, il favore è eccessivo et supera ogni mio merito ${ }^{47}$. Ihm oblag hiermit die Aufsicht über alle Festungen in Ungarn und auch die Stadtbefestigung Wiens. Bereits Anfang Dezember beabsichtigte Don Giovanni Details in einem Gespräch zu klären ${ }^{48}$.

Obwohl es Winter war - in den Briefen berichtet Don Giovanni von großer Kälte (eccessivi fredd $i^{49}$ ) und zugefrorenen Flüssen (non mi sono altrementi mosso $p[$ er $]$ essere sopraggiunto il chiaccio $[=$ ghiaccio $]$ al fiume $\left.e^{50}\right)-$, besichtigte er weit entlegene Festungsanlagen auf ungarischem Gebiet, auch wenn ihm klar war, daß

${ }^{46}$ ASF, M.d.P., filza 5156, fol. 664v-665r: Brief vom 17. 11. 1595 aus Bratislava (Possonia). Bereits zuvor, am 15. Okt., hatte der Großherzog Ferdinand I. den Rückzug seiner Truppen angeordnet, da sie u. a. schlecht behandelt würden (... è stata sempre tanto mal vista et tanto maltrattata); als Grund für seine Entscheidung führt er aber auch den bevorstehenden Winter an; vgl. Firenze e la Toscana dei Medici, Ausstellungskatalog (wie in Anm. 3), 94-95 (Nr. 47.11). In Italien kamen schließlich wohl nur wenige an, wie Erasmo Magno da Velletri berichtet: ... la maggior parte moriron d'infermità ritornando in Italia nel '96 (Firenze, Biblioteca Riccardiana, Ms. 1978, fol. 3r).

${ }^{47}$ ASF, M.d.P., filza 5156, fol. 257r (der Brief ist an den Florentiner Botschafter am Prager Hof gerichtet: All'Ill [ust] re s[igno] re Fran [ces]co Lenzoni Amb[asciato]re del Ser[erenissi]mo GranDuca di Toscana alla Corte Cesarea [vgl. ebd., fol. 262v]]). Gleiches geht auch aus einem anderen Brief Don Giovannis vom 3. 12. 1594 hervor, nun an den Großherzog gerichtet (ebd., fol. 259r): presto andrò a far' reverenza a $S[$ ua $]$ A [ltezza $]$ p [er $]$ ricevere i suoi comandamenti circa le fortificazioni di Vienna et d'altri luoghi d'Ungheria, di che ha voluto la Maestà d[ el] l'Imp [erato] re darmi la sopraintendenza accettata da me conforme a l'ob[b]ligo che me ne impone la cortesissima lettera d[e]lla Maestà Sua (die Bedeutung dieser Briefstelle wurde bereits erkannt: vgl. BALossi [wie in Anm. 1], 33). Hierbei handelte es sich offensichtlich um ein Ehrenamt, da sich der Großherzog wiederholt an Don Giovanni wendet und ihn ermahnt, keine Gelder vom Kaiser anzunehmen; er läßt ihm - zumindest ab dem Sommer 1595 - aus der Staatskasse 1000 Scudi monatlich zukommen; ASF, M.d.P., filza 5153, fol. 44r.

${ }^{48}$ ASF, M.d.P., filza 5156, fol. 259r: für das Zitat s. Anm. 47.

${ }^{49}$ ASF, M.d.P., filza 5156, fol. 309v: Brief Don Giovannis vom 21.1. 1595 (1594 more fiorentino, d. h. nach dem damaligen Florentiner Kalender).

${ }^{50}$ Ebd. 
angesichts der Witterung kaum etwas zu erreichen war (in ogni modo andrò a Comarp $[$ er $]$ stabilire quello che si dovrà fare alla fortificazione, et se bene mi ha detto che in questa stagione non si puó fare cosa alcuna ${ }^{51}$ ). In einer Audienz am 18. Januar 1595 legte er dem Kaiser bereits Zeichnungen für den Ausbau einiger Festungen vor, insbesondere für den Ausbau von Esztergom (dtsch. Gran, Strigonia in den Archivakten $^{52}$ [Abb. 2]): Mostrai poi a S[ua] Maestà il disegno di Strigonia grande fatto con tutte le misure rendendole la ragione $p[\mathrm{er}]$ che l'havevo voluto rappresentare in pianta et non in prospettiva alla Maestà sua, che sapevo che intendeva beniss [im $]$ o il disegno, che consideratolo minutamente mi disse piacerli in quella maniera et che altri che gl'erano stati dati, non li haveva potuti intendere ${ }^{53}$. Offensichtlich hatte Don Giovanni eine Zeichnung angefertigt (oder anfertigen lassen ${ }^{54}$ ), die die Festung im Grundriß und nicht in einer perspektivischen Ansicht darstellte (in pianta et non in prospettiva). Interessant ist hier, daß Don Giovanni Rudolf II. als einen verständigen Kenner von Architekturplänen einschätzte. Sicherlich nicht nur aus Schmeichelei schreibt er, daß der Kaiser Zeichenkenntnisse habe (sapevo che intendeva beniss $[\mathrm{im}]$ o il disegno $\left.{ }^{55}\right)$. Schon zuvor hatte Don Giovanni auf ein Schreiben des Kaisers Bezug genommen und lobend angemerkt, daß dieser eine gute Kenntnis ungarischer Festungen habe: $S[\mathrm{ua}]$ Maestà ragionando seco di fortificare i luoghi suoi piu bisognosi, l'ho trovato bene informato et $d[e]$ l paese et d[e]lle fortificazioni, scrive a me, che prima che si parta, mi voglia parlà d [el]l'opera sua ${ }^{56}$.

Don Giovanni hatte nicht nur Pläne für die Befestigung von Esztergom angefertigt, er legte auch einen Entwurf für den Ausbau der Festung von Komárom (dtsch. Komorn, slowak. Komárno, Comar in den Archivakten ${ }^{57}$ ) vor: Le mostrai poi il disegno d[e]lla fortezza di Comar ${ }^{58}$. Bei der Betrachtung dieses Projektes

${ }^{51}$ ASF, M.d.P., filza 5156, fol. 290r: Brief Don Giovannis vom 7. 1. 1595 (1594 nach dem damaligen Florentiner Kalender); vgl. Gambuti (wie in Anm. 3), 457.

${ }^{52}$ Für die Geschichte einiger Festungen und die Übersetzungen der italienischen Ortsnamen ins Deutsche oder in die ungarische Sprache verweise ich auf die inzwischen etwas veraltete Publikation von Maggiorotti (wie in Anm. 33).

${ }^{53}$ ASF, M.d.P., filza 5156, fol. 316r: Brief Don Giovannis an den Großherzog von Toskana vom 23. 1.1595 ( 1594 nach dem damaligen Florentiner Kalender); vgl. auch Firenze e la Toscana dei Medici, Ausstellungskatalog (wie in Anm. 3), 95 (Nr. 47.12.).

${ }^{54} \mathrm{Zu}$ diesem Aspekt s. u.

${ }_{55}$ Der folgende Satz (... mi disse [...] che altri, che gl'erano stati dati, non li haveva potuti intendere) kann sehr unterschiedlich interpretiert werden: daß Rudolfs Kenntnissen durchaus Grenzen gesetzt waren, da er nicht alle Baupläne lesen konnte, oder - wahrscheinlicher -, daß die Baupläne Don Giovannis sehr viel besser als die anderer Architekten gezeichnet waren.

${ }^{56}$ ASF, M.d.P., filza 5156, fol. 269v: Brief Don Giovannis aus Wien an den Großherzog von Toskana vom 2. 12. 1594.

${ }^{57}$ Zum Namen und zur Geschichte der Festung vgl. Maggiorotri (wie in Anm. 33), 286298, Taf. 65-66.

${ }^{58}$ ASF, M.d.P., filza 5156, fol. 316r (wie oben Anm. 53). 
entdeckte der Kaiser eine Eigenart, d. h. das Fehlen der Ohrenbastionen, ein Charakteristikum, das man bereits bei einigen toskanischen Festungen aus der Zeit Cosimos feststellen kann" ${ }^{59}$ : Le mostrai poi il disegno d[e]lla fortezza di Comar, sopra il quale mosse $S[u a] M[$ aes $]$ tà alcuni dubbi et in part [icola]re p[er]che ai baluardi non fossero fatti gl'orecchioni; et havendo sentita la risposta con gusto di restarne (?) capace et contento, ma molto piu dicendole io che gia io $i[n]$ persona havevo fatto in Comar ficcare i pali et tirare le corde secondo quel' disegno che piacq [ue] a $S[u a]$ Maestà, dicendo che pure sperava di vederla finita ai nostri giorni et che alla fine si terminerebbero tante consulte sopra quella fortezza, et io la avertai che con meno di $150 \mathrm{~m}[\mathrm{ila}]$ tallari si finerebbe $d[e] l$ tutto, et che già vi havevo messo persone intendenti da conducere bene la muragtia $\langle f a b[b] \text { rica }\rangle^{60}$ secondo il disegno et che se non mancheriano $i$ danari et $i$ lavoranti che presto si potrebbe vedere finita ${ }^{61}$. Der Briefstelle kann man entnehmen, daß Don Giovanni trotz der ungünstigen Witterung die Bauobjekte besichtigt und sogar schon begonnen hatte, das Baugelände mit Pfählen abzustecken (havevo fatto in Comar ficcare $i$ pali et tirare le corde secondo quel' disegno). Ebenso geht aus dem Brief hervor, daß der Kaiser den Entwurf umsetzen wollte, auch wenn in der Wahl der Worte bereits erste Zweifel an der Finanzierbarkeit (se non mancheriano i danari) und - angesichts der bevorstehenden Sitzungen zahlreicher Kommissionen - auch Zweifel an der politischen Realisierbarkeit erkennbar werden (sperava di vederla finita ai nostri giorni et che alla fine si terminerebbero tante consulte sopra quella fortezza). In diesem Brief äußert sich Don Giovanni auch bezüglich der Bauspesen, die er mit 150.000 Talern beziffert - einer immens hohen Summe, bedenkt man, daß danach höchstens 20.000 Taler (bzw. fiorini?!) bereitgestellt wurden ${ }^{62}$, manchmal auch erheblich weniger, z. B. nur 6000 Gulden $^{63}$. Da er im Anschluß die Pläne mitnahm (Finalmente ripigliando con sua licenza $i$ disegni), um angeblich auf einem Plan

${ }^{59}$ Ich beziehe mich hierbei auf Festungen wie die fortezza S. Martino in San Piero a Sieve im Mugello-Tal, die von seinem Vater Cosimo I. de' Medici errichtet und anscheinend im Juni 1569 auch vom Großherzog selbst entworfen wurde: Il Granduca Cosimo andò a disegnare e disegnò la Fortezza ch'é sopra a San Piero a Sieve che si chiama S. Martino che gira un miglio in circa e di poi si fondò in ispazio di pochi giorni (SETTIMANNI, Memorie fiorentine III [ = ASF, Ms. 128], fol. 452).

${ }^{60}$ Durchstrichenes und darüber gesetztes Wort.

${ }^{61}$ ASF, M.d.P., filza 5156, fol. 316r/v (s. o., Anm. 53).

${ }^{62}$ ASF, M.d.P., filza 5156, fol. $334 \mathrm{v}$ (Brief vom 21. 2. 1595): ... premendoli sommamente Comar[onio] et Altenburgh, et con molta ragione, $p[$ er $]$ questo dicano havere in essere 20 $m[$ ila $]$ fiorini.

${ }^{63}$ ASF, M.d.P., filza 5156, fol. 290v (Januar 1595), auch fol. 764v (undatierter Brief, aber laut einer zeitgenössischen Anmerkung vom 17.12. 1595): ... pronti li seimila fiorini deputati da $S[$ ua] Maestá p [er] questo serv [izi]o. 
einige Kleinigkeiten $\mathrm{zu}$ vollenden $(p[e r]$ finire alcune cosette), dürften sie noch nicht ganz fertiggestellt gewesen $\operatorname{sein}^{64}$.

Die Audienz nutzte Don Giovanni auch dazu, dem Kaiser seinen Mitarbeiter Claudio Cogorano (1554-1618) ${ }^{65}$ vorzustellen: Introdussi da S[ua] Maestà il Cogorano acciò tanto più guadagnassi la grazia di Cesare, havendole io detto quello che posso d[e]lla sua sussistenza (?) et valor ${ }^{66}$. Die beiden hatten sich anscheinend während des Flandern-Feldzugs kennen und schätzen gelernt.

Es war nicht die einzige Unterredung mit dem Kaiser. Eine weitere fand am 31. Januar $1595^{67}$ und eine dritte einen Monat später, Ende Februar, statt. Don Giovanni schreibt im Brief vom 21. Februar 1595: S[ua] Maestà med[esim]a voleva domani havermi da sep $[$ er $]$ trattarmene ${ }^{68}$. In den nächsten Monaten scheint er nicht mehr beim Kaiser vorgelassen worden zu sein, so sehr er dies wünschte ( $e t$ $i[n]$ voce lo diró meglio a $S[$ ua $]$ Maesta $\left.{ }^{69}\right)$. Erst nach einem Jahr, Ende Januar 1596, hatte er wieder die Ehre einer Audienz - und zwar um dem Kaiser nun seine unmittelbar bevorstehende Abfahrt mitzuteilen. Er schreibt im Brief vom 2. Februar 1596: Hier l'altro fui alla seconda audienza di S[ua] Maestà havendo preso licenza di venire in Italia et sendo restato interamente satisfatto dalla buona cera et amorevoli parole usatemi dalla Maestà sua con maniera di straord [inari] a cortesia ${ }^{70}$. Mehr erfahren wir von dieser Unterredung nicht. In einem Brief zuvor, vom 16. Januar, teilt er immerhin mit, dem Kaiser weitere Entwürfe und Pläne gebracht zu haben: Ho portato un bel disegno di Strigonia a S[ua] Maesta ${ }^{71}$.

${ }^{64}$ ASF, M.d.P., filza 5156, fol. 316v: Finalmente ripigliando con sua licenza $i$ disegni l'uno $p[$ er $]$ finire alcune cosette et l'altro $p$ [er] farlo copiare (Brief vom 23. 1. 1595, d. h. 1594 nach dem damaligen Florentiner Kalender). Die Pläne wurden offensichtlich alle kopiert, um sie z. T. auch nach Florenz an den Großherzog zu senden (s. u., Anm. 112 bzw. im Text). Die Zeichnungen wurden wenig später dem Kaiser zugesandt.

${ }^{65}$ Für eine Biographie vgl. Promis (wie in Anm. 30), 792-799. Vgl. auch F. Linati, Intorno all' Ingegnere Claudio Cogorani - Memorie. Atti e Memorie della RR. Deputazioni di Storia Patria per le Province dell'Emilia, n. s. IV, 1 (1879), 193-221. Später wurde er zum Befehlshaber (capitano) einer Einheit von 100 Mann ernannt.

${ }^{66}$ ASF, M.d.P., filza 5156, fol. 316v (wie oben Anm. 53). Daraufhin wurde Claudio Cogorano als kaiserlicher Ingenieur angestellt: Arrivó Claudio Cogorano chiamato a questo serv [izi] o da $S[u a]$ Maestà $p[e r]$ opera d [e]l Conte Carlo con titolo di ingegnero magg [io]re 100 [fiorini?] il mese di provisione (ASF, M.d.P., filza 5156, fol. 406r).

${ }^{67}$ ASF, M.d.P., filza 5156, fol. 322r (Brief vom 31. 1. 1595): ... oggi dopo magnare sono stato a l'audienza di S[ua] Maestà.

${ }^{68}$ ASF, M.d.P., filza 5156, fol. 334v.

${ }^{69}$ ASF, M.d.P., filza 5156, fol. 684v: Brief Don Giovannis aus Wien vom 2. 12. 1595.

${ }^{70}$ ASF, M.d.P., filza 5156, fol. 747r: Brief Don Giovannis aus Prag vom 2. 2. 1596 (1595 nach dem damaligen Florentiner Kalender).

${ }^{71}$ ASF, M.d.P., filza 5156, fol. 736v: Brief Don Giovannis an den Großherzog von Toskana vom 16.1.1596, 
Während Don Giovanni in seinen Briefen vor allem darauf bedacht ist, seine Rolle in den Unterredungen mit dem Kaiser herauszustreichen, schildern andere Quellen weitere Details. Aus einer anonymen Lebensbeschreibung (Abbozzi d'una vita di Don Giovanni de' Medici), die Giambattista Strozzi (1551-1634) zugeschrieben wird ${ }^{72}$, erfährt man, daß es in den Unterredungen mit dem Kaiser nicht nur um Festungsbaukunde gegangen sei, sondern auch um andere gemeinsame Interessen, wie die Alchimie und Magie: L'Imperatore Ridolfo sentiva consolatione e gusto si grande nel trattar con lui che mentre esso Don Gio [vanni] svernava in Praga, lo voleva tutti i giorni seco e ben spesso senza trattare alcuna cosa grave, quantunque quella maestà ogni volta che lo mandava a chiamare, lo mandasse a chiamare sotto colore di voler colui consultare su le cose della guerra. Trattava il piu di prove di Magia e Negromantia e di meraviglie di Chimica, di che molto si dilettava quell'Imperatore . $^{73}$.

Nachdem Don Giovanni in der letzten Unterredung mit Rudolf II. den Wunsch geäußert hatte, nach Italien zurückkehren zu dürfen, reiste er Ende März 1596 - ohne seine Pläne der ungarischen und Wiener Befestigungsanlagen verwirklicht zu sehen - etwas zermürbt vom höfischen Intrigenspiel und vom ständigen Warten auf die ausstehende Finanzierung seiner Bauprojekte von Wien ab ${ }^{74}$. In Gesellschaft von Gianfrancesco Aldobrandini erreichte er nach etlichen Zwischenstationen und Umwegen über Venedig und Ferrara im April Florenz ${ }^{75}$. Ein zweites Angebot, im Sommer 1596 wieder in derselben Stellung als General am Krieg teilzunehmen, lehnte er dankend $\mathrm{ab}^{76}$.

${ }^{72}$ BNCF, Magl. Cl. IX 124 (einst Strozzi 1152), nun: Cod. 2464, fol. 55r-62v. Es handelt sich um einen Auszug aus einer Lebensbeschreibung Don Giovannis (Abbozzi d'una vita di Don Giovanni de' Medici, c. 8 [= acht Blatt]), die anscheinend für eine Akademie in Livorno verfaßt wurde (vgl. die Anmerkung auf der Rückseite des Faszikels: Per l'Accademia di Livorno). Zu einzelnen Aspekten vgl. Landolfi (wie in Anm. 35), 125-162.

${ }^{73}$ BNCF, Magl. Cl. IX 124 (einst Strozzi 1152), nun: Cod. 2464, fol. 58r. Ungeklärt bleibt allerdings, von wem der Autor der Lebensbeschreibung diese Notizen bekommen hat; er hat sich m. E. weder am österreichischen Hof noch in Ungarn aufgehalten hat (eventuell durch Don Giovanni oder durch den Florentiner Botschafter in Prag?). Zweifellos sind einige Details übertrieben dargestellt.

${ }^{74}$ Der letzte Brief aus Wien ist vom 25. März 1596 (ASF, M.d.P., filza 5156, fol. 753r-755r). Der nächste Brief stammt erst vom 12. Juni 1596 (und wurde von Macerata aus abgeschickt).

${ }^{75}$ ASF, M.d.P., filza 5156, fol. 762r; F. Settimanni berichtet, daß Don Giovanni am 13. April in Florenz eintraf (Notiz dem Diario Mart [?] entnommen): Addi XIII di Marzo 1595 (= 1596), Mercoledi - Fece ritorno in Firenze dall'Ungheria il sig[nor] Don Giovanni de' Medici. - D. Mart. (ASF, Ms. 130, fol. 438v).

${ }^{76}$ Weniger als die Intrigen seitens italienischer Kollegen bzw. Feldherren anderer Nationen hätte es vor allem an der Verschlechterung der diplomatischen Beziehungen zwischen Florenz und dem Kaiserhaus gelegen (insbesondere mit Madrid); vgl. Balossi (wie in Anm. 1), $30,32,34,40$. 
In etlichen Briefen klagt Don Giovanni über ausbleibende Finanzierungen seiner Bauprojekte (Io sono ancora qui aspettando $i$ danari $i^{77}$;...non havendo mai potuto havere $i$ danari destinati da $S[$ ua] Maestà $p$ [er] questo effetto, ne i primi seimila fiorini, ne $i$ cinq $[$ ue] milia altri che $S[u a]$ Maestà scrive havere destinati alla fine di Dicembre ${ }^{78}$ ); gelegentlich zeigt er sich auch erzürnt über das schleppende Voranschreiten der militärischen Aktivitäten ( $p[$ er $]$ che se si perdono queste frontiere, L'Alemagna et l'Italia sta male sendo Vienna tanto male difensibile $\left.{ }^{79}\right)$.

Konkrete Angaben über realisierte Wehrbauten sucht man in den Briefen vergebens - sowohl was die Festungen an der ungarischen Grenze als auch vor allem die Stadtmauern Wiens betrifft. Immerhin schreibt Don Giovanni in einem Brief vom 17. September 1595 an den Großherzog von Toskana, daß mit den aus Böhmen und Mähren erwarteten Geldern erste Befestigungsarbeiten in Komárom in Angriff genommen werden sollten: Ho lasciato ordini e danari $p[$ er $]$ seguitare di restaurare quel' castello (= Komárom), et ho diseg $[$ na $]$ to e stabilito il recinto della nuova fortezza ed i suoi termini et pali in maniera che come venghino i danari che di breve si aspettano di Bohemia et Moravia, vi si poss a cominciare a travagliare et $p$ [er] adesso si farà il recinto di Pali et graticci all'usanza del Paese, et a poco a poco si andrà terrapienando in maniera che con un asseg[namen] to ordinario di non molte mig[liai $]$ a di fiorini l'anno che $S[u a] M[$ aestà $]$ ordini $p[$ er $] q[$ uest $]$ a fabbrica in pochi anni si troverà qui fatto una fortezza la quale senza dubbio sarà il salvamento di questo regno $p$ [er] che come sarà terrapienata et vestito il terrapieno con una camicia di murag $[l i]$ a $p[$ er $]$ conservarlo et difenderlo dall' acqua ardisco di dire ancor' che sia mia creatura che sarà una bella fortezza et cosi pare al Cogorano, il quale mi ha aiutato tirar le corde et disegnarla, et come io sia arrivato a Vienna ne manderò un disegno all'A[ltezza] $V$ [ostra], la quale vi conoscerà qual che cosa che ho imparato et visto con l'esperienza fuori dell'uso commune, ne creda $p[$ er] questo V [ostra] $A[$ ltezza $]$ che $q[$ uell $]$ a mia opp $[$ inio $]$ ne sia un capriccio o bizzarria stravagante, poi che $q[$ uest $]$ o modo et nuova forma di Baluardi piace in maniera al colonnello Orfeo et al Cogorano che ciascuno di loro non li farà mai altrimenti ${ }^{80}$. Es handelte sich offensichtlich um erste Bauarbeiten, zunächst um die Instandsetzung der alten $\operatorname{Burg}(p[$ er $]$ seguitare di restaurare quel castello $)$ und um die Errichtung von provisorischen Erdwällen und Palisadenzäunen, in der Hoffnung, in ein paar Jahren (in pochi anni) eine stattliche Festung bauen zu können (si troverà qui fatto una fortezza la quale senza dubbio sarà il salvamento di questo regno). Nicht ohne Stolz

77 ASF, M.d.P., filza 5156, fol. 287r: Brief vom 30. 12. 1594.

${ }^{78}$ ASF, M.d.P., filza 5156, fol. 289v: Brief vom 7. 1. 1595.

${ }^{79}$ ASF, M.d.P., filza 5156, fol. 335r: Brief vom 21. 2. 1595. - Vgl. auch ebd., 290v-291r (Brief vom 7.1.1595): ... se qua non si muta governo presto presto (sic!) et sia dato ordine alla buona et fedele dispositione d [e]l danaio, che l'Imp [erato]re perderà li stati e l'Italia sarà assai vicina ai pericoli et danni che si possono temere da un inimico (fol. 291r) tanto potente.

${ }^{80}$ ASF, M.d.P., filza 5156, fol. 664r/v: Brief vom 17. 11. 1595. 
fügt er hinzu, daß dieser Wehrbau einzigartig sein werde (fuori dell'uso commune), seine Bastionen neuen Wehrprinzipien (nuova forma di Baluardi) und die ganze Anlage den neuesten Erkenntnissen der Festungsbaukunst (conoscerà qual che cosa che ho imparato et visto con l'esperienza) entsprächen. Weitere Einzelheiten zur Form und dem Aussehen der Festung erfahren wir nicht. Eine Zeichnung (vielleicht einen Grundriß oder eine perspektivische Ansicht?) will er später aus Wien zusenden; dieser Plan ist jedoch verschollen. Möglicherweise lag die Innovation in der Vereinfachung und Reduktion der Bastionen (u.a. dem Weglassen der ,Ohren'). Obwohl dem Kaiser bereits viele Monate vorher, im Januar 1595, Entwürfe vorlagen ${ }^{81}$, war von der neuen Festung im Winter 1595/96 anscheinend noch nichts errichtet worden.

Immerhin scheint die Festung von Komárom später wirklich realisiert worden zu sein (Abb. 3-4). Angesichts der Namen, mit denen die einzelnen Bastionen bezeichnet wurden (Medici, Arciduca, Ungheria, Imperatore, Moravia, Cogorano ${ }^{82}$ ), muß man davon ausgehen, daß Don Giovannis Projekt tatsächlich umgesetzt wurde: sonst hätte man die Bastionen nicht nach den Medici und nach seinem Ingenieur Cogorano benannt! Und in der Tat zeichnet sich die Festung durch eine unterschiedliche und teilweise sehr eigenwillige Form von Bastionen aus, z.T. handelt es sich um ein treppenartig ineinander verschachteltes System von Bastionen verschiedener Größe, sog. rivellini. Es wäre zu überlegen, ob speziell die Form der spitz dem Fluß zugewandten Bastionen, die keine seitlichen Öffnungen für Kanonen (,Ohren') aufweisen, auf Entwürfe Don Giovannis zurückgeht. Es ist aber auch möglich, daß man Don Giovannis Planungstätigkeit auf die unmittelbar vor der Festung liegende Ortschaft beziehen muß. Folgt man den Ausführungen von L. A. Maggiorotti, die auf zeitgenössischem Stichmaterial aufbauen, wäre die Festung prinzipiell Mitte des 16. Jahrhunderts vollendet gewesen, d.h. fast ausschließlich nach Entwürfen von Pietro Ferrabosco erbaut worden ${ }^{83}$. Don Giovanni hätte demnach nur Entwürfe für die Verbesserung der Befestigung - die sog. Instandsetzungsarbeiten - und die Wehrbauten für die unmittelbar davorliegende

${ }^{81}$ S. o., Anm. 58 und 61 sowie im Text. Immerhin begann man im Januar 1596 die Mengen an Baumaterialien zu bestimmen, die man für den Bau der Festung benötigte; ASF, M.d.P., filza 5156, fol. 733v: ... ils [igno]r Sprincistain (= Sprinzenstein) è stato stasera da me, et con occ [asio] ne di havere preso nota di materiali necessarij alla fab $[b]$ rica di Comar (Brief vom 16. 1. 1596).

${ }^{82}$ Vgl. L. ZANGHERI, L'Architettura dell' Impero, in: Il potere e lo spazio - la scena del principe, III. Firenze 1980, 137f. Eine vergleichbare Namensgebung kann man bei der Festung von Györ (deutsch: Raab, italien.: Giaverino) feststellen, bei der ebenfalls zwei Bastionen "Ungheria“ und „Imperatore“, zwei weitere nach den Erbauern „Sforza“ (Sforza Pallavicini war dort seit 1555 Gouverneur gewesen) und „Montesanto“ hießen und eine fünfte nach der Donau benannt wurde („Danubio“), jedoch nie nach dem Namen der ausführenden Baumeister; vgl. MaggiorotTi (wie in Anm. 33), 175 (Abb. 35), 179.

${ }^{83}$ Ebd., 287ff. 
Ortschaft angefertigt, die in der Tat in den Stichen des 16. Jahrhunderts noch mit einer Stadtmauer ohne Bastionen, später aber mit Bastionen dargestellt wird ${ }^{84}$.

Auch bei einer anderen Festung erfahren wir nur von dem Baubeginn: Si è $p[$ ri $] n[$ ci $]$ piato il nuovo forte di Coccheri et $S[$ ua $]$ A[ltezza $]$ hiermatt $[$ in $]$ a che fu il giorno di tutti $i$ santi vi andó con $t[u t]$ ta la sua corte $e^{85}$; mehr aber nicht. Hing die schleppende Bautätigkeit nur von widrigen Umständen ab oder lag es eventuell auch an Don Giovannis Entwürfen, die womöglich nicht überzeugten bzw. nicht den militärischen Anforderungen und Erwartungen entsprachen? Vielleicht waren die geplanten Festungen - zumal angesichts der knappen Kassen - überproportional groß und aufwendig? In diese Richtung scheint eine Äußerung Don Giovannis zu gehen, wonach die Zeit dränge und der Bau großer Festungen zu lange dauern würde bzw. eine unvollendete Festung fast noch gefährlicher sei als eine niemals errichtete: Io non so se il tempo basterà p [er] fare a Comar et Altemburg una fortificazione conveniente a difendersi da una forza turchesca, $p[$ er $]$ che di qui a tempo nuovo mi par poco spazio a tanta Macchina, tanto piu che delli huomini si stenterà a haverne, non sendo a Comar dove stare, se non allo scoperto et anco il non haver i danari pronti et esser impiegati quando ci sono in altro, cagiona sempre dilazione, però vorrei che $V$ [ostra] $S$ [ignoria] cavassi da $S[u a] M[$ aestà] se caso che non ci possi far cosa Reale come converrebbe, vogli che si facci tanto che $i$ soldati possino ripararsi $p[e r]$ adesso et con piu tempo il resto $p[$ er $]$ che il cominciar grande opera et lassarla imperfetta, non so come sia sicur ${ }^{86}$. Nicht ausschließen sollte man, daß der Kaiser auf weitere finanzielle Unterstützungen seitens anderer Herrscher, vor allem des Papstes hoffte (sperando che $S[u a]$ Santità volgerà il suo soccorso d [e]l danaro ${ }^{87}$ ) und deshalb manche Entscheidungen hinausschob.

${ }^{84}$ Diese Annahme scheint eine Bestätigung durch die Planungen von Carlo Tetti (oder: Theti) zu bekommen, der bereits um 1560/70 eine derartige Befestigung der Ortschaft vorschlug, die eventuell erst unter Don Giovanni realisiert wurde.

${ }^{85}$ ASF, M.d.P., filza 5156, fol. 636r: Brief von Cosimo Baroncelli vom 2. 11. 1595, in dem er sich anscheinend auf den Erzherzog Matthias bezieht. Anhand eines handgezeichneten Planes kann man die in der Quelle genannte Festung auf der anderen Donauseite gegenüber von Esztergom, unweit der Mündung des Flusses Gran lokalisieren: dort als Cocchini bezeichnet (Firenze, Biblioteca Riccardiana, Ms. 1978, fol. 14 [tavola 14bis]). Möglicherweise gibt die Zeichnung die bereits errichtete Festung wieder (Abb. 5) und ist daher bald nach 1595/96 zu datieren. Hier (in Auszug) die Legende der Abkürzungen: A = città di Rasciani $; \mathrm{C}=$ Roccha di Stregonia $; \mathrm{D}=$ sepultura del Bego $; \mathrm{E}=$ monte di san Tomasso $; \mathrm{K}=$ paludi; $\mathrm{L}=$ un monasterio disfatto; $\mathrm{M}=$ campo grande; $\mathrm{O}=$ alloggiamento del ser [enissi] mo $D[o n]$ Mateo et sua cavalleria $; \mathrm{P}=$ allogiamento del Arciduca; $\mathrm{R}=$ Ungheri $\mathrm{p}[\mathrm{er}]$ guardia di detto $; \mathrm{S}=$ ponte Maggiore $; \mathrm{T}=$ Cocchini (Festung); $\mathrm{X}=$ due forti uno $\mathrm{p}[\mathrm{er}]$ banda del Danubio con una trinciera con terra tutto il piano $p[e r]$ dove pol venire cavalleria nemica.

${ }^{86}$ ASF, M.d.P., filza 5156, fol. 765v (undatierter Brief, aber laut einer zeitgenössischen Anmerkung vom 17.12.1595).

${ }^{87}$ ASF, M.d.P., filza 5156, fol. 335: Brief vom 21. 2. 1595. 
Um sich ein genaueres Bild des damaligen Geschehens machen zu können, müßte man auch Urteile anderer Zeitgenossen hinzuziehen, doch solche Gegendarstellungen sind rar. Immerhin scheinen die Mitarbeiter Don Giovannis, Claudio Cogorano und ein nicht näher bestimmbarer colonnello Orfeo, mit seinen Lösungen einverstanden gewesen zu sein: ... poi che q[uell $]$ o modo et nuova forma di Baluardi piace in maniera al colonello Orfeo et al Cogorano che ciascuno di loro non li farà mai altrimenti ${ }^{88}$ (das positive Urteil übermittelt uns allerdings Don Giovanni selbst in einem seiner Briefe, weshalb ihm etwas der Charakter eines Eigenlobes anhaftet).

An diesem Punkt möchte ich die Anzahl der Festungen und Bauprojekte bestimmen, an denen Don Giovanni beteiligt war und für die er Zeichnungen anfertigte. In diesem Zusammenhang wird dann auch zu klären sein, wie groß sein Anteil an diesen Bauprojekten war. Stellt man alle von ihm in den Briefen erwähnten Bauzeichnungen zusammen, kommt man auf die stattliche Anzahl von mindestens zwei Dutzend Plänen zu sieben verschiedenen Bauobjekten: d.h. zu den Festungen von Komárom (italienisch Comorra, Don Giovanni nennt sie Comar $^{89}$ ), Mosonmagyaróvár (dtsch. Ungarisch Altenburg, von Don Giovanni Altenburgh ${ }^{90}$ bezeichnet) und vor allem von Esztergom (dtsch. Gran, Strigonia in den Archivakten ${ }^{91}$ [Abb. 2]) und Cocchere bzw. Coccheri ${ }^{92}$ (eine Befestigung auf der gegenüberliegenden Seite der Donau bei Esztergom, [Abb. 5]), ferner auch

${ }^{88}$ S. o., Anm. 80. Aufgrund des Ansehens von Cogorano bedeutete dies viel; vgl. ASF, M.d.P., filza 5153, fol. 36v: Il Cogorano m'è molto lodato da tutti, et sento con molto gusto che ciò che $V$ [ostra] Ecc[ellen] za haveva ordinato per la fortificatione di Comar sia stato da lui approvato.

${ }^{89}$ ASF, M.d.P., filza 5156, fol. 316r: Zitat s. o., Anm. 61 (bzw. entsprechend im Text); und ebenso fol. 412r (s. u., Anm. 108); der italienische Name lautet "Comoriano" (zur Geschichte der Festung und ihrem Namen s. o., Anm. 57).

${ }^{90}$ ASF, M.d.P., filza 5156, fol. 326r (Brief an den Großherzog vom 2. Februar 1595): Hora le posso dire d'havere trattato a lungo con il Dottor Petl conforme a che mi mostró contentarsi $S[u a]$ Maestà, il quale mi dice che domani sarà da me p [er] dirmi quello che l'Imp [erato]re gl'ha commesso, et porterà alcuni disegni di Vienna, Comar et Alte [n] burgh. Zur Geschichte der Festung vgl. Maggiorotri (wie in Anm. 33), 270-274, Taf. 60.

${ }^{91}$ ASF, M.d.P., filza 5156, fol. 634r (Brief Don Giovannis an den Großherzog vom 2. 11. 1595): ... il Cap [itan ]o Claudio Cogorano, che è ingener magg[io]re, et io insieme habbiamo resoluto et terminato che si faccia Cocchere nuovo, et si rassetti il castello et la terra di Strigonia p [er]che quella de Tatiani (?) si deve spianar del tutto et manderó appunto la copia di quel istesso che Noi manderemo o porteremo a $S[u a] M[$ aestá $]$; für weitere Zitate von Briefstellen s. o., Anm. 53 und im Text.

${ }^{92}$ ASF, M.d.P., filza 5156, fol. 634 r (Brief vom 2. 11. 1595): Zitat s. o., Anm. 91; ferner fol. $645 v$ (Brief Don Giovannis vom 9. 11. 1595): Io ho fatto fare una instruttione al Cogorano di quello che si deve fare $p$ [er] hora intorno alla terra e castello di Strig[oni] a et Coccheri nuovo; zur Lage dieser Festung s. o., Anm. 85 bzw. Abb. 5 (im Plan als „T“ eingezeichnet). 
von Noistat $^{93}$ (wohl Neuhäusel, ungar. Érsekújvár, das heutige Nové Zámky, in der Slowakei ${ }^{94}$ ), schließlich zu einem Militärlager (Io ho fatto accomodare tre disegni $d[e] l$ campo sotto Strigonia ${ }^{95}$ ) und einer Donaubrücke (il di seguente io mi sarei partito per Comare insieme con il Marchese [wohl: Borgaut] per vedere dove bisognassi fare il Ponte $p[$ er $]$ andare a Tottes $\left.{ }^{96}\right)$.

Bei diesen Projekten handelt es sich, wie Don Giovanni stets korrekterweise anmerkt, keineswegs immer um eigenhändige Zeichnungen, auch nicht immer um eigene Entwürfe. Oftmals ließ er diese von Spezialisten ausführen oder vollenden, weshalb viele als ein Gemeinschaftswerk anzusehen sind. Ein Mitarbeiter wurde bereits genannt: Claudio Cogorano, mit dem er wiederholt zusammenarbeitete - und anscheinend auch gerne, da er mit ihm noch 1597 die Inselfestungen vor Marseille (das berühmte Château d'If) und in Livorno errichtete ${ }^{97}$. Mit ihm plante er - wie schon gesagt - die Feste von Komárom im November 1595 (Cogorano, il quale mi ha aiutato tirar le corde et disegnarla ${ }^{98}$ ); danach scheint er alle Projekte mit ihm zusammen ausgearbeitet zu haben (u. a. die Festungen Esztergom, in den Akten Strigonia genannt, und Coccher $^{99}$ ). Er vertraute ihm dermaßen, daß er ihm oft die Leitung überließ: Io ho fatto fare una instruttione al Cogorano di quello che si deve fare $p$ [er] hora intorno alla terra e castello di Strig[oni] a et Coccheri nuovo et l'ho fatta lasciare al Sprinzstain ${ }^{100}$ sottoscritta di mano di detto Cogo $[$ ra $]$ no acciò egli che è soprastante delle fabbriche eseguisca quello che egli con mia partecipatione gli lascia scritto in detta istruttione ${ }^{101}$. Mit ihm hat er wohl auch ein militärisches Gesamtkonzept ausgearbeitet, das alle ungarischen Grenzfestungen betraf: ... manderò $i$ disegni di come si doverrebbono assettar $q[$ uest $]$ e Piazze

${ }^{93}$ ASF, M.d.P., filza 5156, fol. 309r/v (Brief vom 21. 1. 1595): Io fui a Noistot (oder: Noistat), veddi quella città et ordinai levarsene la pianta, come si è fatto, vedendosi el[ l] a poterla fare piazza forte, ma con grande spesa, et qualche poco di tempo, il che sarà da me rappresentato a $S[u a]$ Maestà et V[ostra] A[ltezza] in disegno (bereits zitiert von Gambuti [wie in Anm. 3], 457).

${ }^{94}$ Der deutsche Name lautete Neuhäusel, so wie es auch vor 1918 einen ungarischen Namen gab (Éreskújvar).

${ }^{95}$ ASF, M.d.P., filza 5156, fol. 752r: Brief vom 17.2. 1596.

${ }^{96}$ ASF, M.d.P., filza 5156, fol. 591r: Brief vom 11. 10. 1595. Mit Tottes ist offenbar die ungarische Stadt Tata (dtsch. Totis) gemeint.

97 Zum Holzmodell von Cogorano vgl. L. ZaNGHerI, Quattro disegni veri di Don Giovanni de' Medici. Artista. Critica dell'arte in Toscana 3 (1991), 164, Anm. 5; zu seiner Tätigkeit auf der Île d'If s. o., Anm. 30.

${ }^{98}$ ASF, M.d.P., filza 5156, fol. 664r: Schreiben Don Giovannis an den Großherzog von Toskana vom 17. 11. 1595.

${ }^{99}$ S. o., Anm. 91-92.

${ }^{100}$ Es handelt sich um Johann Albrecht Sprinzenstein und Neuhaus (1543-1598), in anderem Kontext Giovan Alb [er] to libero Barone di Spitzenstain et Neuhaus genannt.

${ }^{101}$ ASF, M.d.P., filza 5156, fol. 645v: Brief Don Giovannis vom 9. 11. 1595. 
secondo il parer del Cogorano et mio ${ }^{102}$. Als ,Zeichner' bediente er sich verschiedener Gehilfen, u. a. des Curzio Guglielmi ${ }^{103}$, eines in der Fortifikationskunde bislang unbekannten Spezialisten, der während des Feldzuges 1594 verstarb, des Orazio Grazia Dio, der ebenfalls in Ungarn starb ${ }^{104}$, sowie ferner eines Cap [ita]no Armateo $^{105}$.

Aus einigen Briefen geht aber klar hervor, daß Don Giovanni einige Pläne selbst schuf, z. B. für die Festung von Altenburg ${ }^{106}:$... ho fatto di mia mano l'allogato $[=$ allegato $]$ disegno di Altenburgh, quale le mando ${ }^{107}$. Manchmal scheint es sich dabei allerdings nur um schnell vor Ort angefertigte Arbeitsunterlagen gehandelt zu haben - so im Fall von Komárom (Comar), wie aus einem Brief vom 29. April 1595 hervorgeht: ... et havendo trovato il sito molto diverso da quello [che] mostrano $i$ disegni, ho disegnato come mi pare che possa star bene ${ }^{108}$. Ebenso berichtet Don Giovanni in einem anderen Brief an den Großherzog, daß er einen ersten Entwurf für den Ausbau der Festung von Komárom ausgeführt habe, den er ihm anbei zusendet: Le mando con questa il disegno di Comar fatto da me cosi alla grossa in queste occupationi sendomi morto 2 di fa Orazio Grazia Dio, che mi faceva in queste

${ }^{102}$ ASF, M.d.P., filza 5156, fol. 600r: Schreiben Don Giovannis an den Großherzog von Toskana vom 11. 10.1595.

${ }^{103}$ ASF, M.d.P., filza 5156, fol. 270r (Brief an den Sekretär des Großherzogs, den Cav. Belisario Vinta, vom 2. 12.1594): Curtio Gugelmi ser [enissi] mo signo]re è un gran buon giovane, studioso $d[e]$ lle fortificazioni et $d[e] l$ disegno, et mentre che io son'qua ha servito molto bene, sendosi trovato meco di et notte in tutte le occ [asio] ni (zu seinem Tod s. u. Anm. 104).

${ }^{104}$ ASF, M.d.P., filza 5156, fol. 312r (Brief von M. Giani an den Sekretär des Großherzogs, Belisario Vinta, vom 22.1.1595):S[ua] Ecc[ellen] za [= Don Giovanni] sentirà grand[issi]mo gusto d'haver qualche disegnatore havendone molta necessità dopo la morte di Orazio et poi di Curzio Guglielmi; zum vollständigen Namen ebd., fol. 213: Le mando con questa il disegno di Comar fatto da me cosi alla grossa in queste occupationi sendomi morto 2 di fa Orazio Grazia Dio, che mi faceva in queste cose grand [issi] mo serv [iti] ot si è fatto perdita di un gran buon giovane (Brief vom 24. 10. 1594).

${ }^{105}$ ASF, M.d.P., filza 5156, fol. 348r (Schreiben an den Sekretär des Großherzogs, Belisario Vinta, vom 4. 3. 1595): Il Cap [ita] no Armateo presente et servitore sará racc[omanda] to a $V$ [ostra] $S$ [ignoria] dalle sue buone qualità, si come desidero io che sia $p$ [er] amor mio favorito et aiutato $[. .],. p[$ er $]$ che è huomo franco et di una brava obbedienza senza timore alvero $d[e] l$ la morte, havendo anco assai buono disegno et attitudine a levare piante, come fece ultimamente venendo meco, di quella di Noistot, che messe in disegno giusta e diligente. Zu den in den Briefen genannten Zeichnern zählt wohl auch ein nicht näher bestimmter signor Naldino (ASF, M.d.P., filza 5156, fol. 429r); vermutlich der „capitano“ Ottavio Naldini; vgl. Maggiorotti (wie in Anm. 33), 284.

${ }^{106}$ Korrekterweise: Ungarisch Altenburg bzw. Mosonmagyaróvár in Ungarisch, s. o., Anm. 90.

${ }^{107}$ ASF, M.d.P., filza 5156, fol. 327v: Brief vom 14. 2. 1595.

${ }^{108}$ ASF, M.d.P., filza 5156, fol. 412r: Brief vom 29.4. 1595. 
cose grand [issi $]$ mo serv [iti] ot si è fatto perdita di un gran buon giovane ${ }^{109}$. Er entschuldigt sich ausdrücklich, die Zeichnung selbst angefertigt zu haben, da sein Zeichner gestorben sei. Auch von der Festung Giaverino (Győr in Ungarn) scheint Don Giovanni mindestens Detailpläne geschaffen zu haben, die er nach Florenz sandte, wie aus einer Briefnotiz Alessandro Pieronis hervorgeht: Havendo havuto da Alessandro Paggio dello Ill[ ustrissi] mo et Ecc[ellentissi $]$ mo sig[no]r Don Giovanni un disegno di mano di $S[u a] E[$ ccellenza $]$ del Baluardo che hanno minato $i$ Turchi a Chiaverino, quale me parso molto ben disegnato ep $[$ er $]$ conseguenza degno difarlo vedere a $S[$ ua $]$ A[ltezza $]$ Ser [enissi $]$ ma (d. h. dem Großherzog zeigen) $)^{110}$.

Unglücklicherweise ist nicht eine einzige der einst den Briefen beigefügten Zeichnungen in diesem Aktenkonvolut erhalten geblieben ${ }^{111}$, und dies, obwohl Don Giovanni manchmal bis zu drei Kopien von demselben Plan anfertigen ließ: Io ho fatto accomodare tre disegni d [e]l campo sotto Strigonia, uno p $[$ er $] S[$ ua $]$ Maestà et l'altro $p[$ er] il ser [enissi] mo Arciduca che me l'hanno domandato tutte e due questi Principi, il $3^{\circ}$ et piu diligente $p[$ er $] V[$ ostra $] A[\text { ltezza }]^{112}$.

Um einen Eindruck zu bekommen, wie man sich Don Giovannis Zeichnungen vorstellen muß, kann man Skizzen und Entwürfe aus einem anderen Konvolut in Bologna heranziehen, in dem sich mehrere eigenhändig von ihm ausgeführte Zeichnungen erhalten haben, die größtenteils militärischen Charakters $\operatorname{sind}^{113}$. Es sind Federzeichnungen, die oftmals zahlreiche handschriftliche Anmerkungen und gelegentlich auch kleine Korrekturen aufweisen (Abb. 6). In ihnen hat man vorwiegend Verbesserungs- bzw. Alternativvorschläge zu bereits vorlie-

${ }^{109}$ ASF, M.d.P., filza 5156, fol. 213r: Brief vom 24. 10. 1594 (s. o., Anm. 104); ähnlicher Wortlaut auch in einem Brief vom 3. 12. 1595: ... non ho potuto mandare $i$ disegni di queste piazze che non sono finiti $p[e r]$ la malattia d $[e]$ l mio pittore, hora si fanno $p[e r] S[$ ua $]$ Maestà et $V$ [ostra] A[ltezza] aiutandomi il Cogorano continuo (ebd., fol. 692r).

${ }^{110}$ ASF, M.d.P., filza 853, fol. 10r: Brief von Alessandro Pieroni an den Sekretär Marcello Accolti vom 1.11.1594.

${ }^{111}$ Möglicherweise wurden die Pläne aufgrund ihres Formates und ihrer Bedeutung aussortiert, als die Briefe gebunden wurden, und befinden sich jetzt in einem anderen Bestand: in der sog. Miscellanea Medicea 93 im ASF (vgl. Gambutr [wie in Anm. 3], 457). In der Tat finden sich dort zahlreiche Pläne von ungarischen Festungen, wenn auch wohl von verschiedenen Händen. Es ist auch möglich, daß einige von Giovannis Zeichnungen in den Nachlaß des Architekten Giovanni Pieroni gelangt sind und sich heute in der Biblioteca Universitaria in Bologna befinden (Ms. 935 [B-C]). Dort werden nachweislich einige handschriftliche Aufzeichnungen und auch Skizzen Don Giovannis aufbewahrt: s. u.

${ }^{112}$ ASF, M.d.P., filza 5156, fol. 752r (Brief vom 17.2. 1596): also eine Kopie für den Kaiser in Prag, eine weitere für den Erzherzog Matthias und eine für den Großherzog Ferdinand I. von Toskana.

${ }^{113}$ Bologna, Biblioteca Universitaria: Ms. 935, Teile B-C, bes. Teil B, fol. 99, 102-103, $104-$ 105 und fol. 106-107, die die Festungen von Grosseto betreffen; vgl. ZANGHERI, Quattro disegni (wie in Anm. 97), 158-165, bes. 160 und Abb. auf S. 161 und 163. Vgl. auch DERS., I disegni del Fondo Pieroni in Bologna. Il disegno di architettura 4/7 (1993), 20. 
genden Entwürfen erkennen wollen. Sie entstanden im Zusammenhang mit dem Ausbau der Festung von Grosseto (1594), der südlichsten Befestigung im MediciStaat. Nur eine Zeichnung, eine Festarchitektur, stammt aus einem sakralen Zusammenhang. Es handelt sich dabei anscheinend um einen selbständigen Entwurf Don Giovannis aus dem Jahr 1592 und zwar um einen auffallend guten (Abb. 7), weshalb man seine Entschuldigungen über seine angeblich schwachen Zeichenkünste nicht allzu wörtlich nehmen sollte (disegno di Comar fatto da me cosi alla grossa). Ein Grundproblem scheint darin gelegen zu haben, daß es für einen Fürstensohn wohl nicht standesgemäß war, sich mit dem , Reinzeichnen' von Architekturzeichnungen, einer mechanischen und handwerklichen Betätigung, zu beschäftigen (selbst für das Briefeschreiben hatte man ja seinen Sekretär).

\section{Don Giovanni und die Befestigungen Wiens}

Wie bereits gesagt, war Don Giovanni im Spätherbst 1594 zum obersten Festungsbaumeister Wiens ernannt worden (sopraintendenza d [e]lla fortificazione di Vienna). Hat er wenigstens in diesem Amt etwas erreichen können? Was berichtet er diesbezüglich in seinen Briefen? Angesichts des desolaten Zustandes der spätmittelalterlichen Stadtmauern und aufgrund des Fehlens einer modernen Befestigung scheint in ihm gleich nach seiner Ernennung das Gefühl aufgekommen zu sein, daß jede Baumaßnahme zwecklos sei (Abb. 8). Er schreibt resigniert: ...è ben vero che Vienna è si debole et ha bisogno di tanto tempo et di spesa si eccessiva che io stimerei piu sano consiglio il volgere quella spesa in un ben formato et capitanato esercito $p[\mathrm{er}]$ combattere incontrando il nemico in campagna, non lasciando però di farci qualche riparo ${ }^{114}$. Wie schon bei den ungarischen Festungsprojekten haben finanzielle Engpässe jegliche konkrete Baumaßnahme verhindert: Io nella sopraintendenza datami da S[ua] Maestà sopra le fortificazioni, non ho potuto fare altro che considerare (?) minutamente Vienna di fuori intorno intorno <et di dentro ciascun Baluardo con ogni mag[gio]r diligenza>, non havendo mai potuto havere $i$ danari destinati da $S[$ ua] Maestà $p[$ er] questo effetto, ne i primi seimila fiorini, ne $i$ cinq $[$ ue $]$ milia altri che $S[$ ua $]$ Maestà scrive havere destinati alla fine di Dicembre $e^{115}$. Finanzielle Erwägungen und das Warten auf ausstehende Gelder bestimmen sein Handeln auch noch einen Monat später, Mitte Februar: ... la certezza che p [er] principiare le fabriche saranno in Vienna 10 in $12 \mathrm{~m}$ [ila] fiorini ${ }^{116}$.

Ein weiterer Faktor, der die Planungen eines neuen Bastionssystems von Wien behindert zu haben scheint, war die Existenz bereits bestehender Entwürfe, die erst herangezogen und begutachtet werden mußten: Hora le posso dire d'havere

\footnotetext{
${ }^{114}$ ASF, M.d.P., filza 5156, fol. 257r: Brief vom 30. 11. 1594.

115 ASF, M.d.P., filza 5156, fol. 289v: Brief vom 7.1. 1595.

${ }^{116}$ ASF, M.d.P., filza 5156, fol. 335r: Brief vom 21. 2. 1595.
} 
trattato a lungo con il Dottor Petl [...], il quale mi dice che domani sarà da me $p$ [er] dirmi quello che l'Imp [erato]re gl'ha commesso et porterà alcuni disegni di Vienna $[. . .]^{117}$ che dal s[igno]r Duca di Ferrara sono stati già dati a $S[$ ua $]$ Maesta ${ }^{118}$. Dem Herzog von Ferrara, Alfonso II. d'Este (1533-1597), kam seit seiner Teilnahme an den Balkankriegen von 1566 eine besondere Rolle am Wiener Hof zu. Die vagen Äußerungen ermöglichen es nicht, konkrete Angaben zum Autor dieser Pläne zu machen und auch nicht zur Darstellung. Trotzdem darf man vermuten, daß es sich um Entwürfe gehandelt hat, die das Befestigungssystem Wiens betrafen. Es ist nicht auszuschließen, daß die Briefstelle auf die zwei Jahrzehnte zurückliegenden Planungen von Antonio Lupicini (ca. 1530 - ca. 1598) Bezug nimmt: Dal Segretario di Sua Maestà mi è stato mandato una lettera la quale dicie essere in risposta di quella che $V$ [ostra] $A[$ ltezza $]$ mandò a Sua Maestá, la quale io li mando per che non sono anchora licentiato, e questo è che Sua Maestà glie piac [i] uto dischorre meco alchune cose attenente alla pianta di Vienna, la quale si tratta di agiungervi una fortificatione, pertanto io at $[t]$ endo a servire havendo la mira a quello che mi comandò $V$ [ostra $A[\text { ltezza }]^{119}$. Leider wissen wir auch hierzu kaum etwas Genaues ${ }^{120}$.

Man gewinnt den Eindruck, daß während Don Giovannis Aufenthaltes nichts Konkretes an der Stadtmauer unternommen wurde, denn wenige Jahrzehnte später, während der Regierungszeit von Kaiser Ferdinand II. (reg. 1619-1637), verfaßte Giovanni Pieroni ein Memorandum (Dell'accrescimento della Città di Vienna $\left.{ }^{121}\right)$, in dem er ein neues Befestigungssystem für Wien vorschlägt - nun eine außerhalb der Stadt gelegene Feste, die sich entweder auf der Donauinsel, d.h. im Bereich der späteren Leopoldstadt befinden sollte, oder auf Anhöhen zwischen St. Ulrich (im heutigen Gemeindebezirk Neubau) und dem sog. Wind-

${ }^{117}$ Zur Einfügung (Comar et Alte $[n]$ burgh) s. o., Anm. 90.

${ }_{118}$ ASF, M.d.P., filza 5156, fol. 326r: Brief an den Großherzog von Toskana vom 2. 2. 1595.

${ }^{119}$ Brief an den Großherzog Francesco I. vom 6. 12. 1578 aus Prag (ASF, M.d.P., filza 59, fol. 130); zit. nach Promis (wie in Anm. 30), 655.

${ }^{120}$ Vgl. H. Krause-G. Reichhalter-I. Gaisbauer u. a., Mauern um Wien: die Stadtbefestigung 1529 bis 1857 (Wien archäologisch 6). Wien 2009, 30ff. Wie Maggiorotti nachweist, war auch Francesco Benigno um 1575 mit der Erneuerung der Stadtbefestigung Wiens beschäftigt; vgl. MAGgIOROTTI (wie in Anm. 33), 180.

${ }^{121}$ Bologna, Biblioteca Universitaria, Ms. 935 [B], fol. 81-86: Der in Schönschrift verfaßte Text ist Kaiser Ferdinand II. gewidmet (Alla Sacra Cesarea Maestà di FERDINANDO SECONDO, Invittissimo sempre Augusto) und von Giovanni Pieroni unterschrieben (vollständiger Text im Anhang). Zur Person Pieronis vgl. L. FraTI: Due ingegneri militari poco noti. Archivio storico italiano 50 (1912), insbes. 103 ff.; vgl. auch L. ZANGHERI, Giovanni Pieroni e Baccio del Bianco a Praga e nell'Impero, in: Centri e periferie del barocco. Il barocco romano e l'Europa, hrsg. von M. FAgiolo-M. L. Madonna (Akten des Kongresses „Roma, l'Italia e l'Europa“, Rom 1987). Roma 1992, 505-515. 
mühlgrund (im heutigen Gemeindebezirk Mariahilf) ${ }^{122}$ (Abb. 11). Dennoch scheint Giovanni Pieroni (1586-1654) in seinem Memorandum an einem Punkt auf die Arbeiten von Don Giovanni Bezug zu nehmen, indem er schreibt: ... per la quale trovò che si restò di forteficarvi l'anno 1597, ben che ne fusse fatto et approvato un Disegno ${ }^{123}$. Vermutlich hatte man bereits im Zusammenhang mit Don Giovannis Planungen begonnen, eine Zitadelle auf der Donauinsel zu errichten, die Arbeiten dann aber unterbrochen. Weshalb die Arbeiten unterbrochen wurden, verschweigt Giovanni Pieroni.

Bekanntlich hatte Kaiser Rudolf II. Prag zu seiner Hauptstadt gemacht. Dies könnte der Grund für die Unterbrechungen der Befestigungsarbeiten in Wien gewesen sein. Als Kaiser Matthias (1612-1619) die Hauptstadt 1618 nach Wien zurückverlegte, stellte sich die Frage der Erneuerung des veralteten Befestigungssystems der Donaustadt wieder. Bis dahin scheint man kaum etwas am Wehrsystem Wiens verändert zu haben, selbst am Unterhalt der Bastionen scheint man gespart zu haben (dies geht u. a. aus einem weiteren Bericht ${ }^{124}$ und aus dem Brief C. Baroncellis von 1619 hervor $\left.{ }^{125}\right)$.

Die Wahl von Giovanni Pieroni zum Festungsbaumeister dürfte nicht zufällig gewesen sein, war er doch Sohn des Florentiners Alessandro Pieroni (ca. 15501607), der sich als Ingenieur und getreuer Mitarbeiter Don Giovannis einen Namen gemacht hatte; außerdem war Giovanni Pieroni Patenkind Don Giovannis. Es ist daher nicht ausgeschlossen, daß die dem Memorandum beigefügte Zeichnung, die hier zum ersten Mal veröffentlicht wird (Abb. 9), zum Teil auf Don Giovannis Planungen zurückgeht, auch wenn dessen Entwürfe zu dem Zeitpunkt gut 20 Jahre zurücklagen. Zudem befindet sich das Memorandum noch heute in einem Konvolut von Unterlagen ${ }^{126}$, in dem neben zahlreichen anderen

${ }^{122}$ Bologna, Biblioteca Universitaria, Ms. 935 [B], fol. 82r: Uno è nell'Isola contigua allà Città, tra la piccola acqua del Danubio e la prossima grande. L'altro è alla parte opposta, cioè non passando l'acqua, inverso $S[$ an $]$ to Ulrico e verso il luogo detto Il mulino à vento (vollständiger Text im Anhang).

${ }^{123}$ Bologna, Biblioteca Universitaria, Ms. 935 [B], fol. 82v. Siehe Anhang.

${ }^{124}$ Bologna, Biblioteca Universitaria, Ms. 935 [B], fol. 88r: ... il Baluardo del Scioten (= Schotten), quale va in rovina et è forza di incamiciarlo, perche altrimenti in occas [ion] e presto sarebbe disfatto e dannoso alla Piazza (der zusammen mit einem Colonello Coriers verfaßte Bericht ist nicht datiert, aber wohl auch in der Regierungszeit Kaiser Ferdinands II. entstanden).

${ }^{125}$ Cosimo Baroncelli schreibt Don Giovanni in einem Brief vom 5. Januar 1619: Le rendo humiliss [imament] e grazie della gazzetta e quanto alle nuove di Bohemia s' intende che le cose vanno maliss [imament] e p [er] l'inperadore e si dubita di Vienna dove $S[$ ua] $M[$ aestà] dicono cerca di fortificare a piu potere (ASF, M.d.P., filza 5147, fol. 261r): Ersichtlich ist, daß der Kaiser, nun Ferdinand II., beabsichtigte, die Stadt durch ein besseres Befestigungssystem zu schützen.

${ }^{126}$ Bologna, Biblioteca Universitaria, Ms. 935, Teile B-C; s. o., Anm. 113. 
Entwürfen von Giovanni Pieroni die einzigen bislang bekannten Zeichnungen Don Giovannis aufbewahrt werden (Abb. 6-7). Das ist sicher kein Zufall.

Angesichts der Tatsache, daß sich derzeit kein eigenhändiger Entwurf Don Giovannis von seinen Festungsprojekten in Ungarn und Wien nachweisen läßt, ist es nicht leicht, ein abschließendes Urteil über seine Leistungen als Festungsbaumeister zu fällen. Die einst den Zeichnungen beigefügten Begleitbriefe, die ich als Quellen für diesen Beitrag herangezogen habe, können nur zu einem Teil diesen Verlust ersetzen. Wie aber aus diesen Briefen hervorgeht, fiel Don Giovanni durch seine innovativen Ideen (Verzicht auf Ohrenbastionen) wie auch durch seine anschauliche Darstellungsweise auf. Sowohl seine italienischen Mitarbeiter als auch Kaiser Rudolf II., obwohl an den nördlichen Festungsbau gewöhnt, waren von seinen Lösungen überzeugt. Seine Innovationen fanden Anerkennung. Seine Herkunft stellte dabei kein Hindernis dar (sie spielte nur bei der Besetzung der ranghohen Ämter im Heer eine Rolle ${ }^{127}$ ). Auffallenderweise hat er ungern Zeichnungen selbst fertiggestellt, sondern diese Tätigkeit lieber Mitarbeitern überlassen. Von seinen Projekten sind anscheinend nur wenige umgesetzt und realisiert worden: die Feste Komárom und eine Donaubrücke. Viele seiner Pläne scheinen hingegen für spätere Entwürfe herangezogen worden zu sein: so seine Planung für die Befestigung Wiens, die wahrscheinlich Giovanni Pieroni verwandt hat. Aufgrund der ihm hier nachgewiesenen, leider heute verschollenen Zeichnungen und seiner damaligen Anerkennung kann Don Giovanni ohne Zweifel als ein fachkundiger und kompetenter Spezialist in Sachen Festungsbau bezeichnet werden. Da er aufgrund seines adligen Standes und seines Ranges keine Bezahlung annehmen durfte ${ }^{128}$, kann er als Architekturdilettant bezeichnet werden - im Sinne des althergebrachten Begriffes, wonach Adlige nicht für Geld arbeiteten, sondern aus innerer Verpflichtung bzw. aus Vergnügen (dies erklärt vielleicht auch, warum er Zeichnungen selten selbst ausführte).

Das hier untersuchte Aktenkonvolut - die aus Ungarn 1594-1596 stammende Korrespondenz Don Giovannis - bezieht sich vor allem auf seine Planungstätigkeit, nicht auf die Umsetzung seiner Projekte bzw. auf den Bau der Festungen. Der Bau dauerte meistens länger und fiel daher ausschließlich in die

${ }^{127}$ Diese Stellen sollten möglichst von Familienmitgliedern des Kaiserhauses oder wenigsten von Hofchargen besetzt werden; vgl. ASF, M.d.P., filza 5156, fol. 333v (Brief an den Großherzog von Toskana vom 21. 2. 1595): Se bene S[ua] Maestà quando concesse la patente haveva disegno che di casa d'Austria saria il Generale et il luogotenente generale, tutt [avvi] a che sendosi in istato da non poter effettuare et continuare tale disegno, quando pure il Generale sij di casa d'Austria et il luogotenente di piu bassa cond [itio] ne pensa non dover $S[u a]$ ecc [ellen] za farmi difficoltà masime nella persona d[e]ls[ignor] Conte Carlo di Mansfelt oltre le qualità et esperienza sua militare sendo anche Principe d [ el] l'Imperio.

${ }^{128}$ S. o., Anm. 47. 
Zeit nach seiner Abreise aus Ungarn; nur wenige seiner Festungsentwürfe wurden umgesetzt. Aus den Briefen Don Giovannis erfahren wir daher nichts zum Bau der Festungen. Es bedarf hierfür anderer Quellen. Die bereits zitierte anonyme Lebensbeschreibung Don Giovannis, nach seinem Tode 1621 als Nachruf verfaßt, bezieht sich hierauf: Diede Don Gio [vanni] il disegno co'l quale fu fabbricato in Ongharia Castel Nuovo, il quale quanto fusse bene inteso ce l'ha dimostrato il Conte di Buequoi (= Charles Bonaventure de Longueval, Graf von Bucquoy?) essendoli ultimam [en] te stato intorno con un fiorito esercito molti mesi invano ${ }^{129}$. Demnach soll nicht nur die Festung von Komárom, sondern auch die von Nové Zámky (einstige deutsche Ortsbezeichnung: Neuhäusel [Abb. 10]) in der heutigen Slowakei unter Verwendung seiner Pläne realisiert worden sein. Dies kann nicht ganz stimmen, denn der Bau wird dem Architekten Ottavio Baldigara zugeschrieben. Allerdings zog sich die Vollendung der Festung über viele Jahrzehnte hin, noch um 1663 waren nur drei Bastionen fertiggestellt. Auch in diesem halbfertigen Zustand hielt die Festung mehreren Belagerungen stand - und dies wahrscheinlich vor allem wegen der vorgelagerten Erdwälle. Vermutlich stammten diese von Don Giovanni, zumal in den Dokumenten auch der Name von Cogorano auftaucht, der für den Bau solcher Außenforts berühmt war ${ }^{130}$.

Da viele Pläne Don Giovannis - u. a. die für die Stadtmauern Wiens - nicht umgesetzt und die Festungen Ungarns in den Kriegswirren der letzten Jahrhunderte oftmals verändert wurden, ging der Ruhm Don Giovannis als eines kaiserlichen Festungsbaumeisters schnell verloren. Geblieben sind uns die wenigen Notizen, die ich in diesem Beitrag vorgestellt habe. Aber die Hoffnung besteht weiterhin, daß - nach dem Prinzip Stein auf Stein - auch in diesem Fall sich weiteres Material finden wird.

${ }^{129}$ BNCF, Magl. Cl. IX 124 (einst Strozzi 1152), jetzt: Cod. Nr. 2464, fol. 60r. Da - wie bereits bemerkt - einige Details in diesem Nachruf übertrieben dargestellt sind, darf man dies auch hier vermuten.

${ }^{130}$ ASF, M.d.P., filza 5156, fol. 457v (Brief vom 22. 6. 1595): Il Cogorano è tornato da Comar a disegnare <uno $>z$ forti $[\mathrm{er}]$ il ponte (dieses Außenfort ist auf zeitgenössischen Stichdarstellungen zu sehen). 


\section{ANHANG}

Konzept von Giovanni Pieroni (1586-1654) zum Bau eines befestigten Stadterweiterungsgebietes vor den Toren Wiens: Alternativprojekte für den Bereich der heutigen Leopoldstadt bzw. auf der Anhöhe zwischen St. Ulrich (Neubau) und dem sog. Windmühlgrund (Mariahilf).

Bologna, Biblioteca Universitaria, Hs. 935 [B], fol. 81r(-86): Alla Sacra Cesarea Maestà di FERDINANDO SECONDO, Invittissimo sempre Augusto

\section{Dell'accrescimento della Città di Vienna (Abb. 11)}

\section{[fol. 82r] Sacra Cesarea Maestà, Sig[no]re Clement [issi]mo}

Due sono i siti attorno alla Città di Vienna, ove per aggrandirla pare che possa con ragione estendersi l'accrescimento: Uno è nell'Isola contigua alla Città, tra la piccola acqua del Danubio e la prossima grande. L'altro è alla parte opposta, cioè non passando l'acqua, inverso S[an] to Ulrico e verso il luogo detto Il Mulino à vento.

De quali il primo per fortificare è meglio del secondo. Il secondo per fare abitazione è piu comodo del primo. Per il che ho fatto Disegni per ambedue, quali humiliss[imamen $]$ te appresento alla Ces[are $]$ a $M[$ aes $]$ tà $V[$ ost $]$ ra.

Nell'Isola stimo esser necessario che una volta si fortifichi, per esser quella unico e certo assicuramento dei soccorsi, che per il Danubio grande si possono havere a Vienna, a pena possibili di poter mai essere impediti, siche se ella fusse assediata, sarebbe però sempre aperta dalla parte dell'acqua, e però un esercito non potrebbe da una parte serrarla, ne due facilmente astringerla; ma si bene potrebbero le due cittadi soccorrersi scambievolmente per la vicinanza, et in caso di perdita d'una resterebbe salva l'altra, per la divisione che haverebbero: Si che tra esse sarebbe partecipazione e separazione ad arbitrio.

Oltra ciò il sito di tale Isola, come piano, libro da ogni predominio e scarso di terreno per un nemico, che volesse trincerarvisi $[$ fol. $82 \mathrm{v}]$ è attissimo e proportionato naturalmente a ricever fortificatione, ancor che gli vengano opposte tre particolari eccettioni:

- La prima è di esser sottoposto alle inondationi del fume, per la quale trovò che si restò di forteficarvi l'anno 1597, ben che ne fusse fatto et approvato un Disegno; ma peró vedesi che la Olanda tutta e tanti altri paesi e piazze patiscono per natura tale eccettione, e con tutto cio esser fortificate, poiche rimediato a q[ue] lla con l'arte quanto bisogna, diviene poi il luogo piu forte e quasi inespugnabile, come molti esempi dimostrano.

- La seconda eccettione è del non vi esser fondo per cavarvi le cortine, ma ponendosi i primi piani elevati sopra il suolo, che vi è hora, la terra che si cavasse per i fondamenti alzando le strade, e quello che il tempo suol fare alzare tutte le cittadi farebbe restare profondità bastante per le cantine: Però se al principio del fabbri- 
care si ponesse un livello universale, al pari del quale tutti posassero la soglia, ne resultarebbe facilmente rimedio bastante.

- Ma la terza eccettione, che è dell'Aria non buona, pare di minor consideratione, perchè empiendosi la città di abitanti l'aria farebbe buona, come à miei giorni ne ho veduto l'esempio nella città di Livorno.

E se alcuno avesse consideratione alla troppa vicinanza [fol. 83r] delle Caccie della $M[$ aes] tà $V[$ ost $]$ ra rimedia interamente il Levare il Commatio [?] della Città a q[ue]lla parte, non vi essendo necessario. Sichè le eccettioni appariscano remediabili et i requisiti, che ha tal sito importantissimi. Per il che ho formato un Disegno per tale Isola, esprimendo il concetto che haverei, quando si risolvesse la $M[$ aes] tà V [ost] ra di edificar la Città in quella.

Questo fo che arrivi da un acqua all'altra per i fini predetti e fa fronte su'l Danubio grande, la quale lo guarda e spazza per ogni verso: Peró non si è possuto circondare con meno di nove interi Baluardi et una Piattaforma, restando la parte verso Vienna, quando si volesse o bisognasse, da potersi chiudere proportionatamente. Cosi i Baluardi riescono assai buoni, la Città di forma bella, l'acqua che haverebbe il fosso la renderebbe molto forte et il porto disegnatovi apporterebbe grand'utile e comoditá; ma le altre particolarità si potrebbero considerare, quando tal sito fusse approvato.

Ma tre sono per mio parere i motivi principali, per i quali si stima doversi far l'aggrandimento della Città dall'altra parte, cioé contiguamente a Vienna.

- Il primo di necessità per occupare e comprendere il luogo alto che è vicino a Vienna, parendo forza di liberarla dal soggiacere [fol. 83v] a quello.

- Il secondo di convienenza per allontanare le prime offese dal Palazzo della M[aes]tà V [ost] ra.

- Il terzo di comodità per haver da fabbricare le abitazioni in sito buono, bello e vicino, anzi unito.

Per i quali fini non è dubbio, che tal luogo non è altrove che inverso il detto mulino a vento, ove comincia una campagna piana, alta e levata sopra il basso, nel quale corre il fume della Vienna; e si estende verso $S[$ an $]$ to Ulrico e par grande spatio dietro, e passando per dove corre il fumicello che viene da Ernola ${ }^{131}$ circonda et arriva ad un ciglio lungo, che sta à Cavaliere del piano del Danubio fuori della porta nuova ${ }^{132}$.

Per il qual sito ho formato due disegni, ciascuno de quali comprende gl'altri predetti, copre il Palazzo di M[aes] tà V[ost] ra, il quale non di meno havrebbe facile uscita della Città, facendosi solamente un corritore dentro alle mura, che arrivasse

${ }^{131}$ Es handelt sich offenbar um den Alserbach und Hernals (im XVII. Wiener Gemeindebezirk).

${ }^{132}$ Das sog. Neutor befand sich im nordwestlichen Abschnitt der Stadtmauern, unweit des Donauufers. 
insino al Baluardo di Carintia, cioè a canto alla porta; e vi è piazza molto comoda per adattarvisi i quartieri delle case, nelle forme disegnate o altre.

Di questi uno che è il maggiore con dieci interi Baluardi s'accosta a Vienna rincontro al Baluardo di Carintia da una parte e rincontro al Baluardo detto Nuovo dall'altra ${ }^{133}$, formato cosi ad intentione, di arrivare nel predetto piano alto a vedere $e$ dominare dall'una e dall'altra parte i luoghi bassi [fol. 84r] cio è del fiume della Vienna da un lato e del piano del Danubio dall'altro, comprendendo et il mulino detto e S[an] to Ulrico. Che però estendendosi questo per tutto il Piano alto, che è attorno a Vienna con predominio sopra tutte le parti basse, pare molto conveniente a tal sito.

Ma perche possono haversi circa di esso due considerationi, una è che sia forse troppo grande e ricerchi però troppo Presidio per guardia e troppa gente per difesa; l'altra che si accosti troppo ad una valle disrupata, lunga e profonda, che è dove passa il fumicello di Ernolz ${ }^{134}$, la quale essendo capace di alloggiare coperto gran numero di gente, potrebbe di là un nemico in una notte aprir Trincera ed arrivare insino al fosso della fortificat $[i o]$ ne che gli fusse troppo vicina: però quando o tanta grandezza non piacesse, o la Tanaglia accennata nel disegno, che arrivi a scovare detta Valle, non fusse reputata rimedio bastante e non sene trovasse altro migliore. Ho fatto l'altro Disegno minore con solo otto interi Baluardi, il quale comincia ove il primo incontro al baluardo di Carintia, ma temina rincontro al Baluardo del Soten ${ }^{135}$, libero cosi dalle predette due eccettioni e comprendente non di meno il d $[\mathrm{ett}]$ o Mulino e $S[$ an $]$ to Ulrico, ma non arriva a scoprire il piano basso del Danubio, si che senza qualche eccettione non si adatta facilmente un recinto in tal sito.

[fol. 84v] Ma in ciascuno di questi ho havuto le seguenti avvertenze, cioè

- Che il il primo intero Baluardo verso il fume Vienna, essendo in sito basso, scovi quella rupe alta e scoscesa, che è sotto il Mulino a vento e spazzi tutto il piano sotto di essa.

- Che la salita, che deve fare il recinto per arrivare all'alto del detto mulino sia compresa tra punta e punta de Baluardi, accio che i fianchi loro possino vedersi e difendersi scambievolmente le fronti de loro Baluardi.

- Che un Baluardo che è il terzo, sia nell'alto ove[ro] di sopra il basso del fiume Vienna e la pendenza che vi descende, al che non bastava il secondo Baluardo, ben che sia nell'alto in parte cioè mezo.

- Che una piccola Valle, che è dietro al Borgo de Capuccini ${ }^{136}$, nella quale corre unacqua ${ }^{137}$, passi per una cortina e vicina al fianco, accio che $i$ due Baluardi che la difendono siano di sito ugualmente alti tra loro.

${ }^{133}$ Gemeint sind die Kärntner- und die Neutorbastei.

${ }^{134}$ Der schon erwähnte, aus Hernals herabfließende Alserbach.

135 Gemeint ist die Schottenbastei.

${ }^{136}$ Es handelt sich offenbar um den unteren Abschnitt der heutigen Neustiftgasse im VII. Wiener Gemeindebezirk, der noch im frühen 19. Jahrhundert Kapuzinergasse genannt wurde.

${ }^{137}$ Gemeint ist vermutlich der Ottakringerbach. 
- Che il recinto finisca verso Vienna con mez[z]i Baluardi, acciò che per se stesso habbia tutte le difese necessarie e non vi siano fianchi volti alla offesa di Vienna.

- Che tali mezi Bal[uar]di siano veduti per fuori e per dentro dal Bal[uar]do, al quale s'avvicinano, acciò che la fortezza di Vienna gli sia di aiuto di fuori e di predominio di dentro, in caso di bisogno.

[fol. 85r] E per tal ragione ancora, che tutto il recinto nuovo sia aperto per dentro inverso Vienna, si che ella sia sempre una sicura ritirita, e che perdendosi il nuovo, sia quanto ad essa, come non perduto altro che un pezzo di Campagna.

E finalmente che tutte le faccie de Baluardi, i Fianchi, le Cortine e le Difese siano uguali respettivamente tra loro, quanto è possibile, accio che tutte le sue parti siano ugualmente forti e ricerchino la medesima pratica e medesimi tiri per la difesa, il che nel minor disegno riesce interamente e nel maggiore con poca differenza. Ma le misure particolari delli recinti, dell'area loro et il calculo prossimam [en] te della spesa le ho notate in piedi de i propri Disegni.

Questo è quanto la povertà del mio ingegno ha saputo produrre, benchè con ogni industria, per servire la Ces[are] a Maestà V[ost]ra mio Clement [issi] mo Sig[no]re alla q[ua]le però appresento un altro Disegno che è della Pianta di Vienna e del sito attorno ad essa, misurato $p[e r]$ le mie forze con ogni giustezza, senza alcun concetto dentrovi, acciochè altri più intendenti, doppo haver censurati e corretti i miei errori al che humil[ issimamen] te e volentieri gli sottopongo, possino disegnarvi altre forme meglioriper maggior servitio della $M[$ aes] tà $V$ [ost] ra, alla quale humiliss[imamen]te inchinandomi prego dal Sig $[$ no] re Dio lunghiss [imament] felicitadi.
Della Ces[are] a Maestà V[ost]ra
Humiliss $[$ im $]$ o e fedeliss [im $]$ o servo
Giovanni Pieroni

\section{ABBILDUNGSNACHWEIS:}

Abb. 1: $\quad$ Palazzo di Cerreto Guidi (Firenze).

Abb. 2, 3, 5: www. istitutodatini.it/biblio/images/en/riccard/1978/htm/elenco.htm.

Abb. 4: $\quad$ nach: MAGgiorotTi (wie in Anm. 33), Taf. 66.

Abb. 6, 7: nach: ZANGHERI, Quattro disegni (wie in Anm. 97), Abb. auf S. 163 und 159.

Abb. 8, 10: Archiv des Verfassers.

Abb. 9: Bologna, Biblioteca Universitaria, Cod. 935 “C”, Plan Nr. 60bis (mit Genehmigung der Biblioteca Universitaria di Bologna; es ist keinerlei Art von Reproduktion oder Vervielfältigung gestattet).

Abb. 11: Bologna, Biblioteca Universitaria, Cod. 935 “B”, fol. 81r (mit Genehmigung der Biblioteca Universitaria di Bologna; es ist keinerlei Art von Reproduktion oder Vervielfältigung gestattet). 


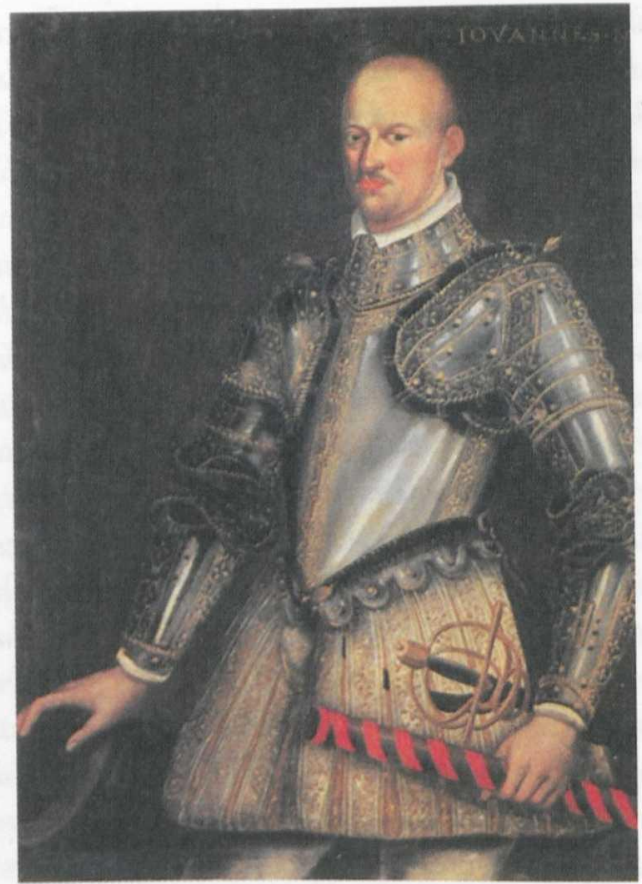

Abb. 1: Porträt von Don Giovanni de' Medici (1567-1621), im Palazzo di Cerreto Guidi (Firenze), eventuell Kopie nach Justus Sustermans oder Santi di Tito (Gemälde am Rahmen unten stark beschnitten)

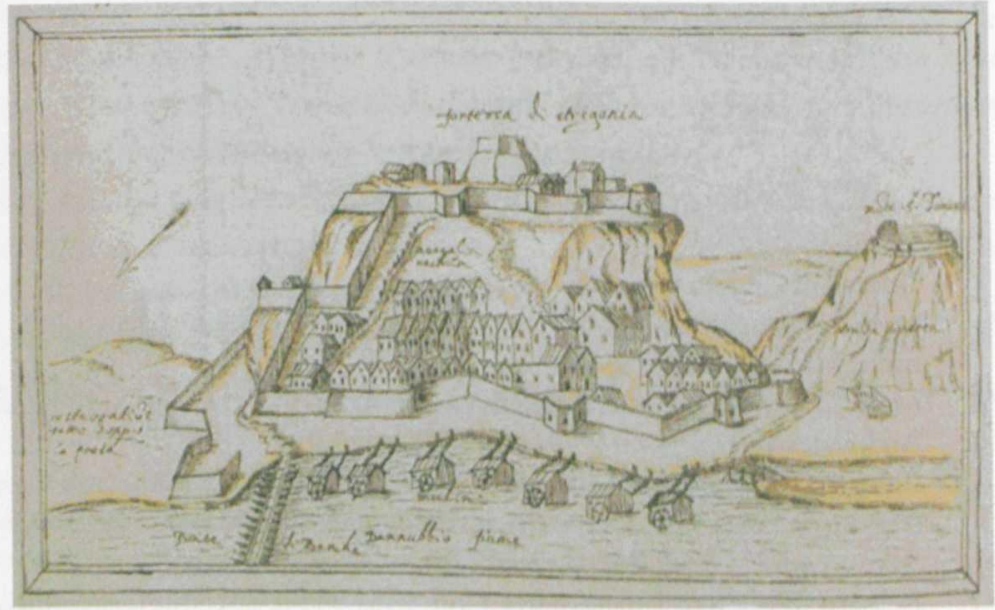

Abb. 2: Ansicht der Befestigungen von Esztergom („Strigonia“) in Ungarn, $120 \times 205$ mm; Erasmo Magno da Velletri: Imprese delle galere toscane, ca. 1597-1616 (Florenz, Biblioteca Riccardiana, Ms. 1978, fol. 14v) 


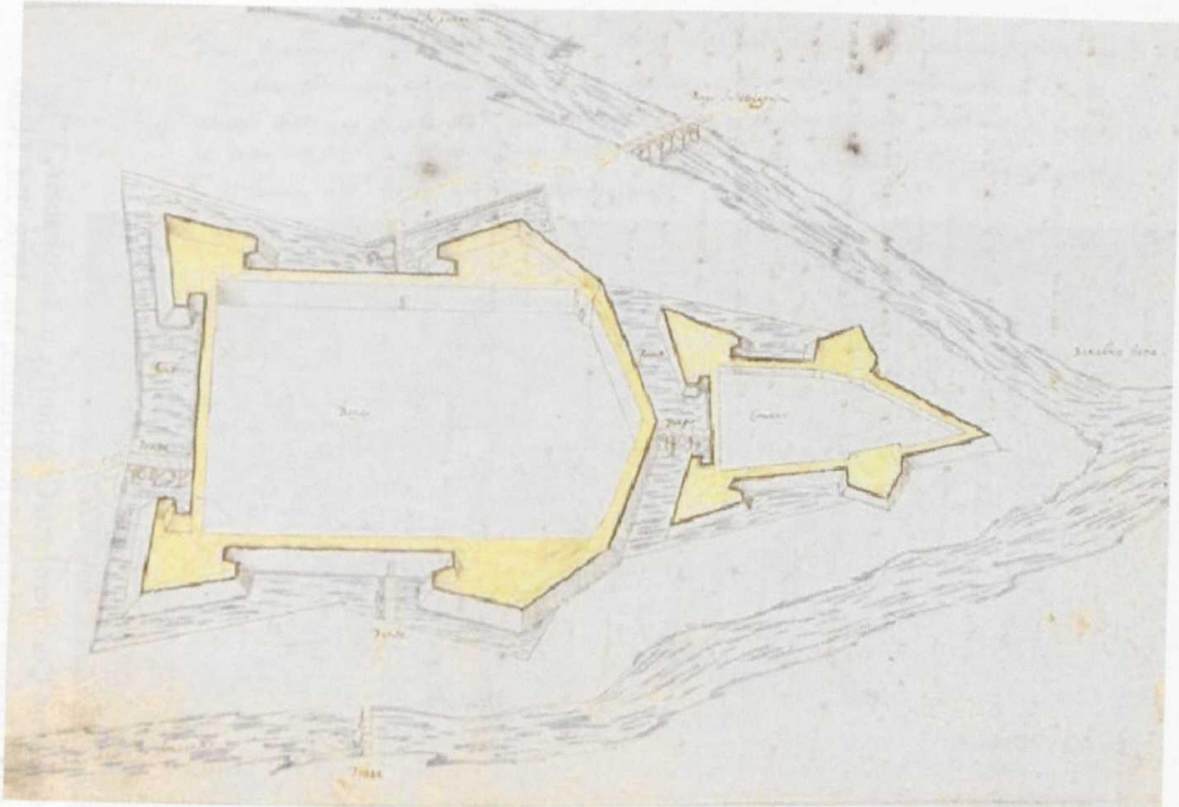

Abb. 3: Grundriß der Festung Komárom („Comar“) in Ungarn, mm $330 \times 210$;

Erasmo Magno da Velletri: Imprese delle galere toscane, ca. 1597-1616 (Florenz, Biblioteca Riccardiana, Ms. 1978), fol. 12r

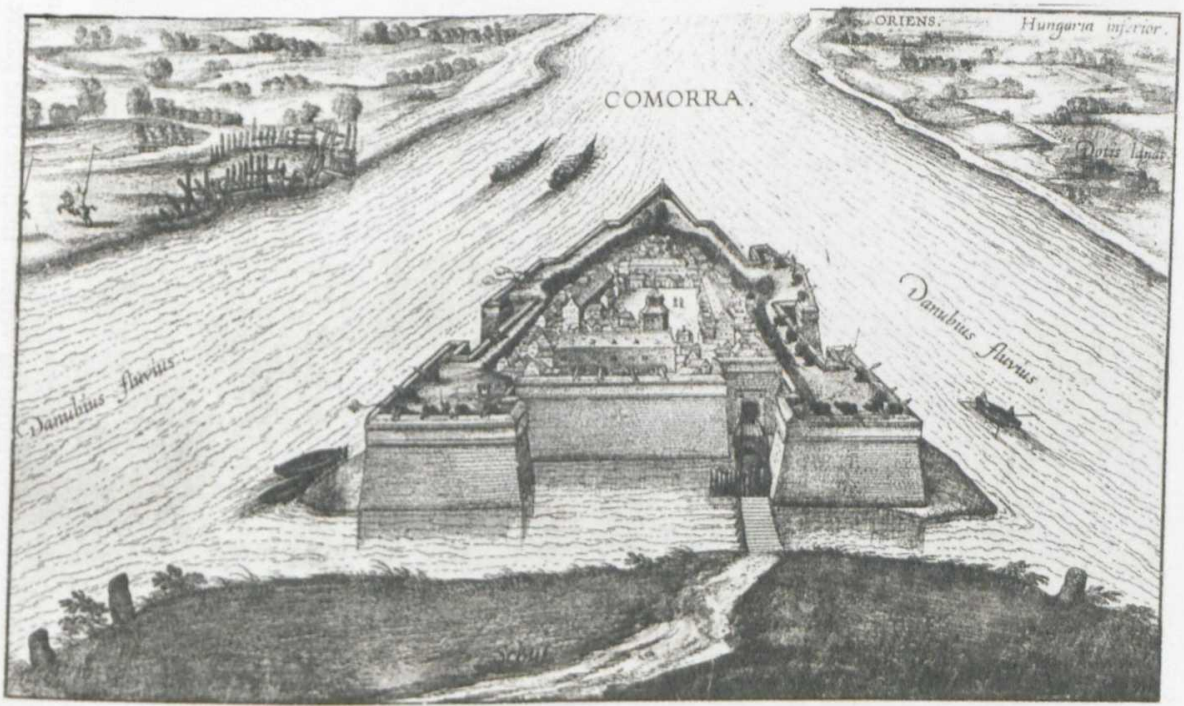

COMORRA - VEDUTA PROSPETTICA NEL DISEGNO DI G. HOEFNAGEL (1595)

Abb. 4: perspektivische Ansicht der Festung Komárom („Comorra“) in Ungarn; Kupferstich von Jacob Hoefnagel 


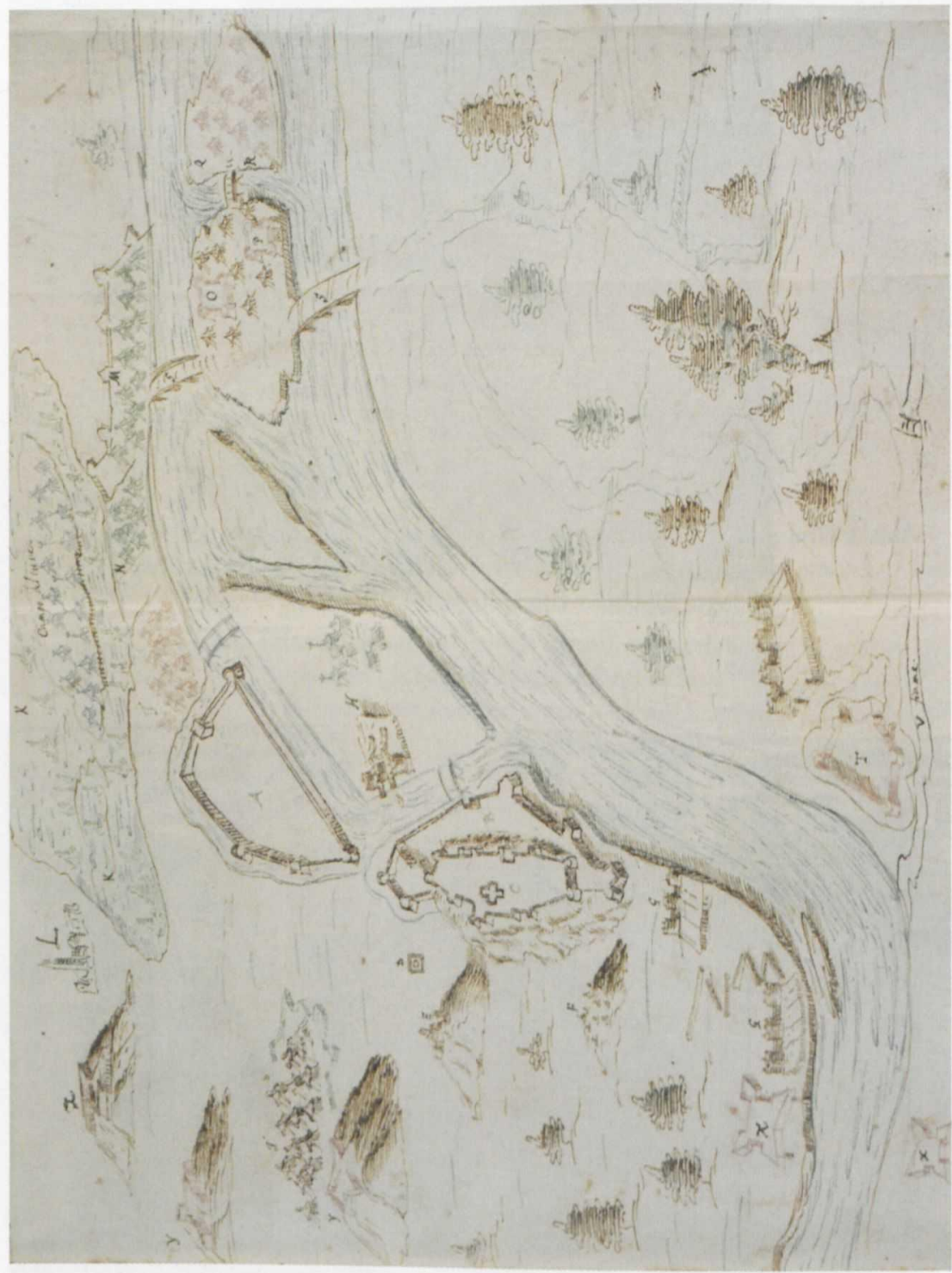

$\stackrel{5}{\circ}$

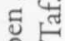

可

ป

m.

군

ن)

青

.

अ च

กำ

言

鸟

0 돈

ㄷㅇ을

잉

들

出 1

岁

峕

E "

ธू ส

5 题

픈

क्ष

है

욜

के

E

응

ㅂ.

缶

ช

잉

है

돈

คㅇำ

ชै

号㝳

哥

in

से 


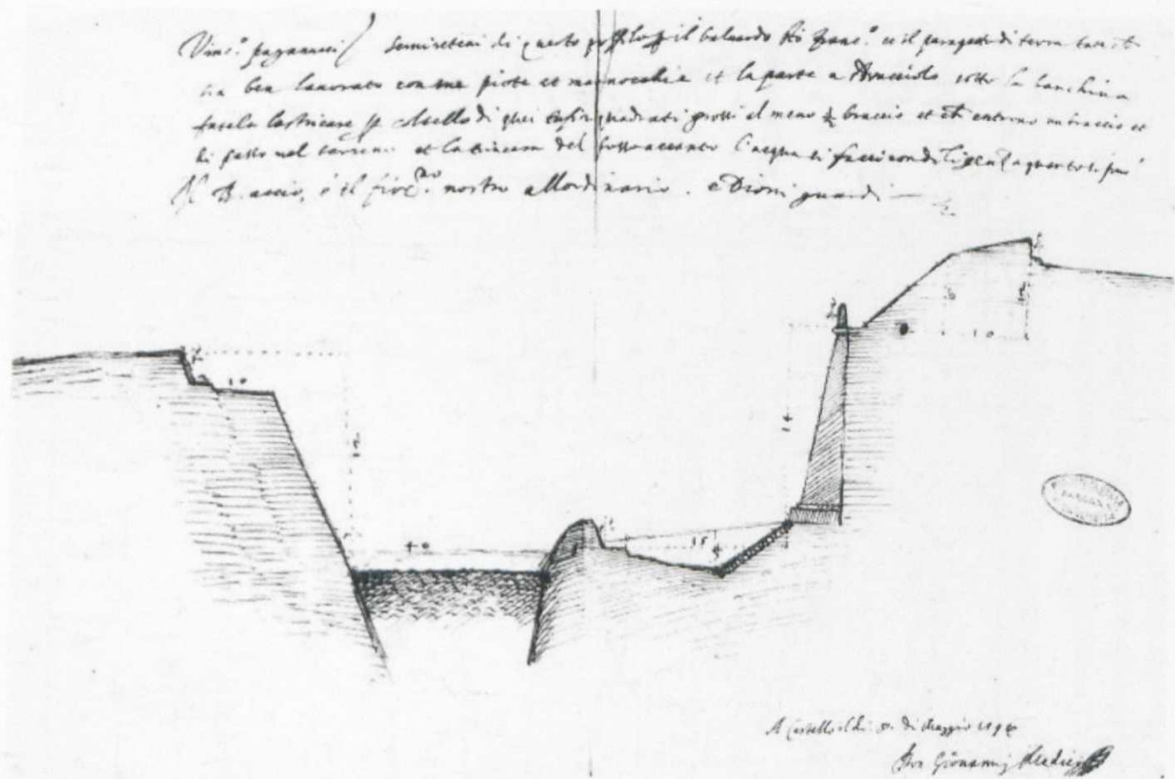

Abb. 6: Don Giovanni de' Medici: Erneuerung der Stadtmauern von Grosseto (Mai 1594):

Federzeichnung, $408 \times 278$ mm (Bologna, Biblioteca Universitaria, Cod. 935 „B“, fol. 106v-107r)

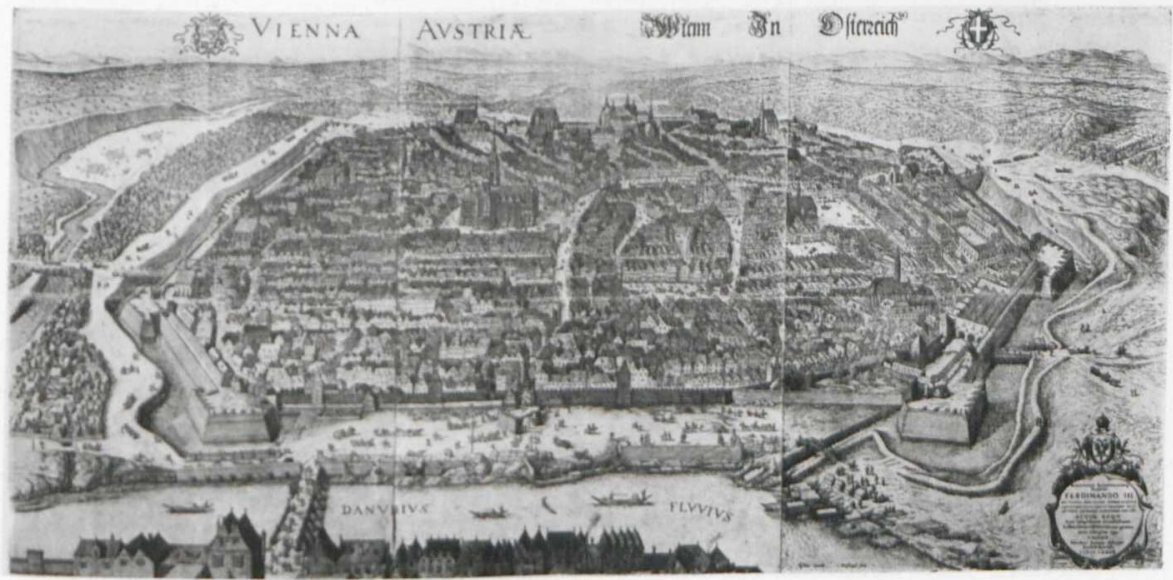

Abb. 8: Perspektivische Ansicht der Stadt Wien; Kupferstich von Jacob Hoefnagel, 1609 


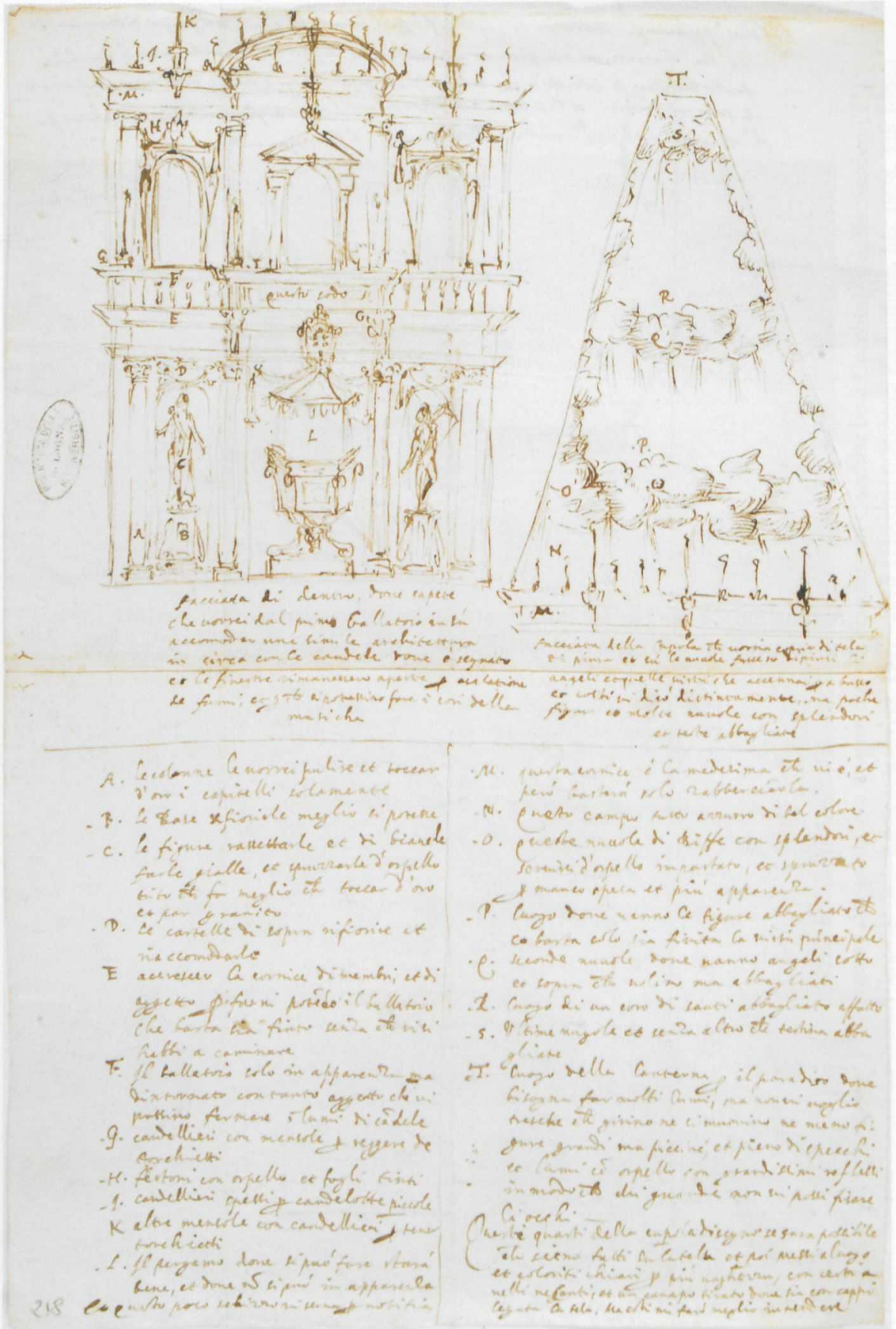

Abb. 7: Don Giovanni de' Medici: Entwurf für Festaufbauten im Baptisterium von Florenz anläßlich der Taufe von Leonora de' Medici, der Tochter des Großherzogs Ferdinando I., 1592 (Bologna, Biblioteca Universitaria, Cod. 935 „B“: $407 \times 275 \mathrm{~mm}$ ) 

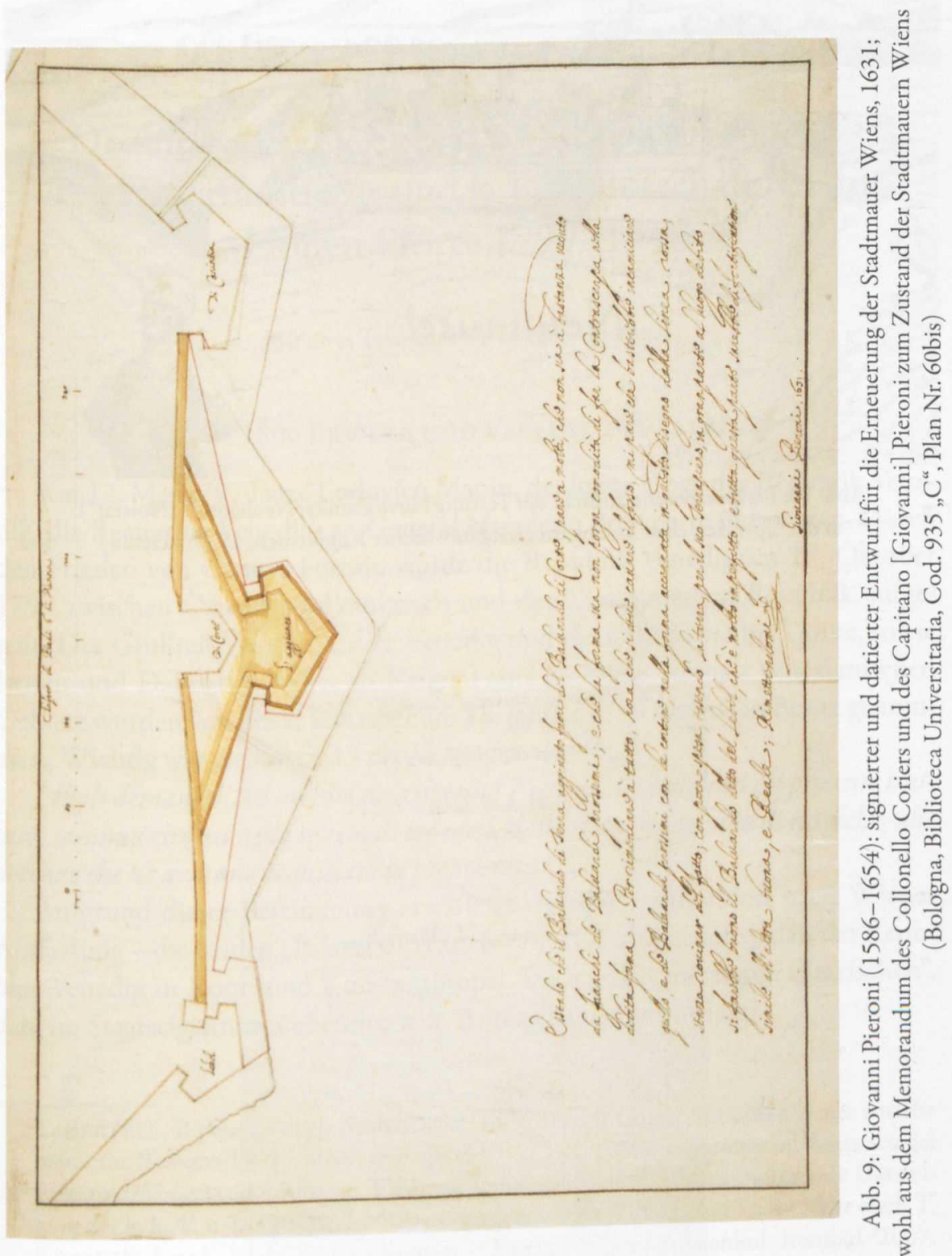


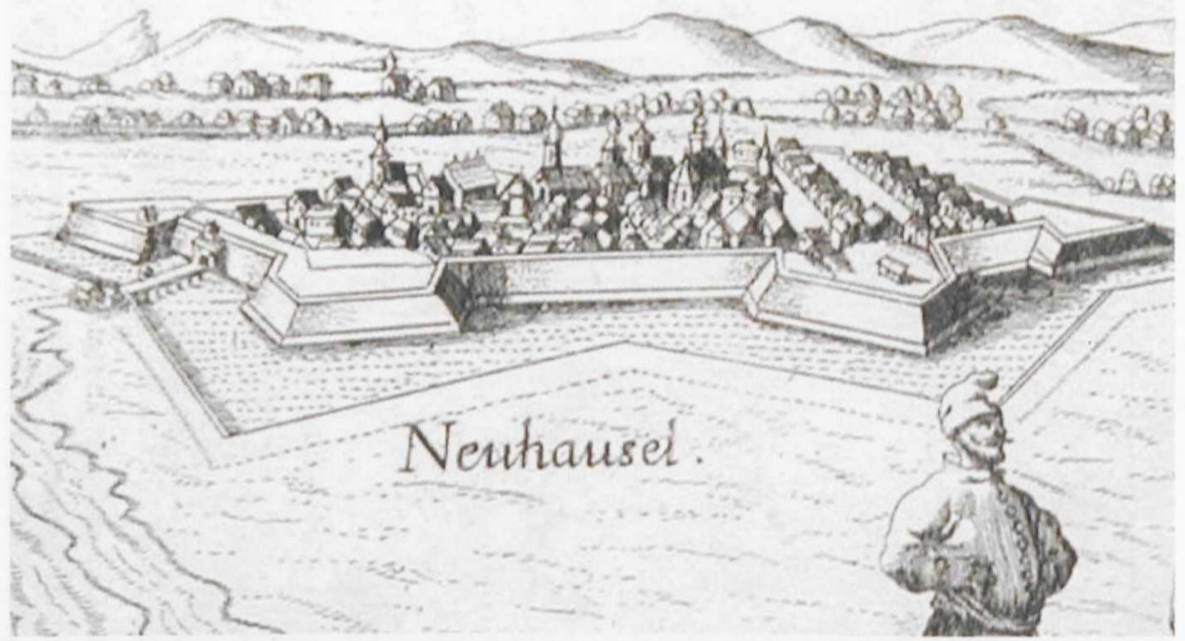

Abb. 10: Perspektivische Ansicht der Festung Nove Zamky/Neuhäusel („Noistat“) in der Slowakei, einst in Ungarn (zeitgenössischer Kupferstich, 17. Jh., Detail)

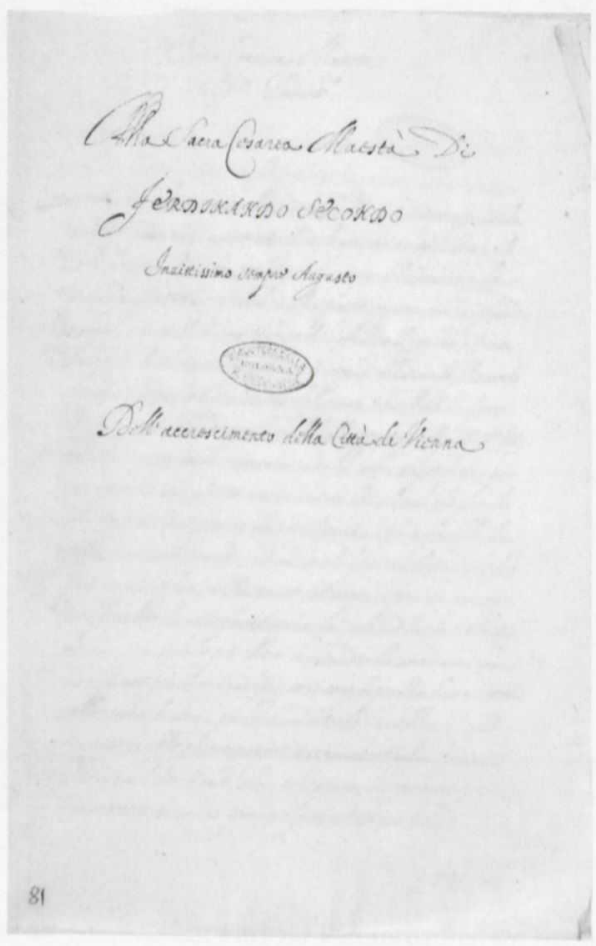

Abb. 11: Giovanni Pieroni (1586-1654): Dell'accrescimento della Citta di Vienna, ca. 1620/30

(Bologna, Biblioteca Universitaria, Cod. 935 „B“, fol. 81r: Titelblatt des Memorandum) 